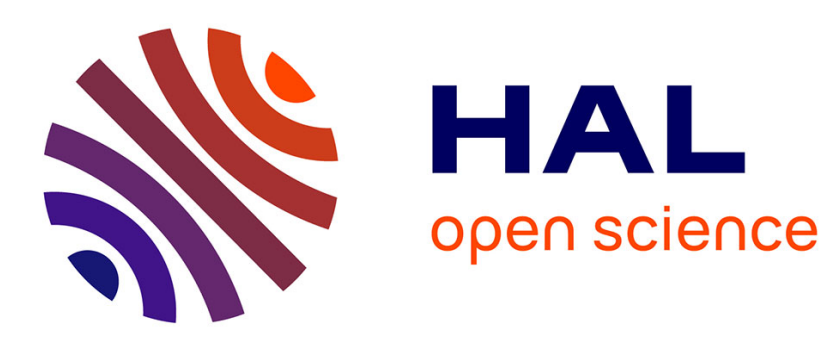

\title{
Penalized contrast estimator for adaptive density deconvolution
}

Fabienne Comte, Yves Rozenholc, Marie-Luce Taupin

\section{To cite this version:}

Fabienne Comte, Yves Rozenholc, Marie-Luce Taupin. Penalized contrast estimator for adaptive density deconvolution. Canadian Journal of Statistics, 2006, 34 (3), pp.431-452. 10.1002/cjs.5550340305 . hal-00016489

\section{HAL Id: hal-00016489 \\ https://hal.science/hal-00016489}

Submitted on 5 Jan 2006

HAL is a multi-disciplinary open access archive for the deposit and dissemination of scientific research documents, whether they are published or not. The documents may come from teaching and research institutions in France or abroad, or from public or private research centers.
L'archive ouverte pluridisciplinaire HAL, est destinée au dépôt et à la diffusion de documents scientifiques de niveau recherche, publiés ou non, émanant des établissements d'enseignement et de recherche français ou étrangers, des laboratoires publics ou privés. 


\title{
Penalized contrast estimator for adaptive den- sity deconvolution
}

\author{
Fabienne Comte, Yves Rozenholc and Marie-Luce Taupin
}

Key words and phrases: Adaptive estimation. Density deconvolution. Model selection. Penalized contrast. Projection Estimator.

MSC 2000: Primary 62G07. Secondary 62G20.

\begin{abstract}
The authors consider the problem of estimating the density $g$ of independent and identically distributed variables $X_{i}$, from a sample $Z_{1}, \ldots, Z_{n}$ where $Z_{i}=X_{i}+\sigma \varepsilon_{i}, i=1, \ldots, n, \varepsilon$ is a noise independent of $X$, with $\sigma \varepsilon$ having known distribution. They present a model selection procedure allowing to construct an adaptive estimator of $g$ and to find non-asymptotic bounds for its $\mathbb{L}_{2}(\mathbb{R})$-risk. The estimator achieves the minimax rate of convergence, in most cases where lowers bounds are available. A simulation study gives an illustration of the good practical performances of the method.
\end{abstract}

Déconvolution adaptative de densité par contraste pénalisé.

Résumé : Les auteurs considèrent le problème de déconvolution c'est-à-dire de l'estimation de la densité de variables aléatoires identiquement distribuées $X_{i}$, à partir de l'observation de $Z_{i}$ où $Z_{i}=X_{i}+\sigma \varepsilon_{i}$, pour $i=1, \ldots, n$, où les erreurs $\sigma \varepsilon_{i}$ sont de densité connue. Par une procédure de sélection de modèles qui permet d'obtenir des bornes de risque non asymptotiques, ils construisent un estimateur adaptatif de la densité des $X_{i}$. L'estimateur atteint de façon automatique la vitesse minimax dans la plupart des cas, que les erreurs ou la densité à estimer soient peu ou très régulières. Une étude par simulation illustre les bonnes performances pratiques de la méthode.

\section{INTRODUCTION}

We observe $Z_{1}, \cdots, Z_{n}, n$ independent and identically distributed (i.i.d.) copies of $Z$ in the model

$$
Z=X+\sigma \varepsilon
$$

where $X$ and $\varepsilon$ are independent random variables, with unknown density $g$ for $X$, known density $f_{\varepsilon}$ for $\varepsilon$, and known noise level $\sigma$. In this model, we aim at estimating the density $g$ without any prior knowledge on its smoothness, using the observations $Z_{1}, \cdots, Z_{n}$ and the knowledge of the convolution kernel $\sigma f_{\varepsilon}(\cdot / \sigma)$. The parametrer $\sigma$ is only estimable under more restrictive conditions on $g$, such as a lower bound on its Fourier transform. However, under the usual conditions on $g$ (as in the currrent paper), $\sigma$ has to be known. We refer to Butucea and Matias (2005) for the problem of the estimation of $\sigma$ as well as for results about density deconvolution when $\sigma$ is unknown in such a model.

In density deconvolution, two factors determine the estimation accuracy. First, the smoothness of the density to be estimated, $g$, and second the smoothness of the errors density, the worst rates of convergence being obtained for the smoothest errors density. Indeed, due to the independence of $X$ and $\varepsilon$, the density $h$ of $Z$ is $h(\cdot)=g *\left(\sigma f_{\varepsilon}(\cdot / \sigma)\right)$, where $*$ denotes the convolution product, and if $f_{\varepsilon}$ is very smooth then so is $h$, the density of the observations and thus it is difficult to recover $g$. 
In this context, we consider two classes of errors: first the so called ordinary smooth errors with polynomial decay of their Fourier transform and second, the supersmooth errors with Fourier transform having an exponential decay.

Most previous results concern kernel estimators and densities $g$ to be estimated belonging to Hölder or Sobolev classes with known order $s$. One can cite among others Carroll and Hall (1988), Devroye (1989), Fan (1991a, b), Liu and Taylor (1989), Masry (1991), Stefanski and Carroll (1990), Zhang (1990), Koo (1999), Cator (2001).

Smoother densities $g$ with exponential decrease of their Fourier transform, have been first considered by Pensky and Vidakovic (1999), Butucea (2004) and Butucea and Tsybakov (2004). The latter study the sharp optimality (in a minimax sense) by using non adaptive kernel estimators and provide an adaptive estimator in some special case. The former is the first paper dealing with adaptivity in a general context. This first adaptive estimator is a wavelet estimator, that achieves the minimax rates when $g$ belongs to some Sobolev class, but that fails in reaching the minimax rates when both the errors density and $g$ are super smooth. Let us mention also Pensky (2002) for the estimation of irregular functions and Fan and Koo (2002) who consider wavelet estimators for densities belonging to Besov spaces. Lastly, analogously to Hesse (1999), Delaigle and Gijbels $(2004 a, b)$ study adaptive methods using cross validation and bootstrap methods in the kernel context.

In the spirit of Barron et al. (1999), we build an adaptive estimator $\tilde{g}$, constructed by model selection, and more precisely by minimization of a penalized contrast function. We show that $\tilde{g}$ is adaptive in the sense that its construction does not require any prior smoothness knowledge on $g$ and that its rate of convergence is the minimax rate of convergence (up to some logarithmic factor) in all cases where lower bounds are previously known, that is in most cases. More precisely, we establish non-asymptotic bounds for its integrated quadratic risk that ensure an automatic trade-off between a bias term and a penalty term, only depending on the observations and on $\sigma f_{\varepsilon}(\cdot / \sigma)$.

The estimator automatically achieves the best rate obtained by the collection of non-penalized estimators when the (unknown) optimal space is selected, exactly or sometimes within a negligible logarithmic factor. In all cases where lower bounds are available, this best rate is the minimax rate of convergence. In particular, when both the density and the errors are super smooth $(\delta>0$ and $r>0$ in $\left(\mathrm{A}_{2}^{\varepsilon}\right)$ and $\left(\mathrm{R}_{1}^{X}\right)$ below), our adaptive estimator significantly improves the rates given by the adaptive estimator built in Pensky and Vidakovic (1999) whereas both adaptive estimators have the same rate in the other cases (see Section 4.3).

The paper is organized as follows. In Section 2, we present the assumptions and the estimators. In Section 3 we give upper bounds for the $\mathbb{L}_{2}(\mathbb{R})$-risk of the estimator, when the smoothness of $g$ is known, and study the optimality in a minimax sense of the resulting rates. In Section 4 , we give upper bounds of the $\mathbb{L}_{2}(\mathbb{R})$-risk of the penalized minimum contrast estimator $\tilde{g}$ when no prior knowledge on the smoothness of $g$ is used. The theoretical results are illustrated by a simulation study in Section 5, and all the proofs are gathered in Section 6.

\section{CONSTRUCTION OF THE ESTIMATORS}

For $u$ and $v$ in $\mathbb{L}_{2}(\mathbb{R}), u^{*}$ denotes the Fourier transform of $u, u^{*}(x)=\int e^{i t x} u(t) d t, u * v$ is the convolution product, $u * v(x)=\int u(t) v(x-t) d t,\|u\|=\left(\int|u|^{2}(x) d x\right)^{1 / 2}$, and $\langle s, t\rangle=\int s(x) \overline{t(x)} d x$.

\subsection{Model and Assumptions}

We require that $f_{\varepsilon}$ belongs to $\mathbb{L}_{2}(\mathbb{R})$ and that for all $x \in \mathbb{R}, f_{\varepsilon}^{*}(x) \neq 0$. We consider that:

$\left(\mathrm{A}_{1}^{X, \varepsilon}\right)$ : The sequences $\left(\varepsilon_{i}\right)_{i \in \mathbb{N}}$ and $\left(X_{i}\right)_{i \in \mathbb{N}}$ are sequences of independent random variables. 
The smoothness of $f_{\varepsilon}$ is described by the following assumption.

$\left(\mathrm{A}_{2}^{\varepsilon}\right): \quad$ There exist nonnegative numbers $\kappa_{0}, \kappa_{0}^{\prime}, \gamma, \mu$, and $\delta$ such that $f_{\varepsilon}^{*}$ satisfies $\kappa_{0}\left(x^{2}+1\right)^{-\gamma / 2} \exp \left\{-\mu|x|^{\delta}\right\} \leq\left|f_{\varepsilon}^{*}(x)\right| \leq \kappa_{0}^{\prime}\left(x^{2}+1\right)^{-\gamma / 2} \exp \left\{-\mu|x|^{\delta}\right\}$

Only the left-hand side of $\left(\mathrm{A}_{2}^{\varepsilon}\right)$ is required for upper bounds whereas the right-hand side is useful when we consider lower bounds and optimality, in a minimax sense, of our estimators.

When $\delta=0$ in $\left(\mathrm{A}_{2}^{\varepsilon}\right)$, the errors are usually called "ordinary smooth" errors, and they are called "super smooth" when $\mu>0$ and $\delta>0$. Indeed densities satisfying $\left(\mathrm{A}_{2}^{\varepsilon}\right)$ with $\delta>0$ and $\mu>0$ are infinitely differentiable. The standard examples for super smooth densities are the following: Gaussian or Cauchy distributions are super smooth of order $\gamma=0, \delta=2$ and $\gamma=0, \delta=1$ respectively. For ordinary smooth densities, one can cite for instance the double exponential (also called Laplace) distribution with $\delta=0=\mu$ and $\gamma=2$. Although densities with $\delta>2$ exist, they are difficult to express in a closed form. Nevertheless, our results hold for such densities. Furthermore, the square integrability of $f_{\varepsilon}$ and $\left(\mathrm{A}_{2}^{\varepsilon}\right)$ require that $\gamma>1 / 2$ when $\delta=0$.

By convention, we set $\mu=0$ when $\delta=0$ and we assume that $\mu>0$ when $\delta>0$. In the same way, if $\sigma=0$, the $X_{i}$ 's are directly observed without noise and we set $\mu=\gamma=\delta=0$ in this case.

Although, slower rates of convergence for estimating $g$ are obtained for smoother error density, those rates can be improved by some additional regularity conditions on $g$. Those regularity conditions are described as follows.

$$
\begin{aligned}
& \left(\mathrm{R}_{1}^{X}\right): \quad \text { There exists some positive real numbers } s, r, b \text { such that } g \text { belongs to } \\
& \qquad \mathcal{S}_{s, r, b}\left(C_{1}\right)=\left\{\psi / \int_{-\infty}^{+\infty}\left|\psi^{*}(x)\right|^{2}\left(x^{2}+1\right)^{s} \exp \left\{2 b|x|^{r}\right\} d x \leq C_{1}\right\} \\
& \left(\mathrm{R}_{2}^{X}\right):
\end{aligned}
$$

The smoothness classes described by $\left(\mathrm{R}_{1}^{X}\right)$ are classically considered both in deconvolution and in "direct" density estimation, with $\mathcal{S}_{s, 0, b}\left(C_{1}\right)$ known as Sobolev classes. The densities satisfying $\left(\mathrm{R}_{1}^{X}\right)$ with $r>0, b>0$ are infinitely many times differentiable, admit analytic continuation on a finite width strip when $r=1$ and on the whole complex plane if $r=2$. The densities satisfying $\left(\mathrm{R}_{2}^{X}\right)$, often called entire functions, admit analytic continuation in the whole complex plane (see Ibragimov and Hasminskii (1983)).

Subsequently, the density $g$ is supposed to satisfy the following assumption.

$$
\left(\mathrm{A}_{3}^{X}\right): \text { The density } g \in \mathbb{L}_{2}(\mathbb{R}) \text { and there exists } M_{2}>0 \text {, such that } \int x^{2} g^{2}(x) d x<M_{2}<+\infty \text {. }
$$

Assumption $\left(\mathrm{A}_{3}^{X}\right)$ which is due to the construction of the estimator, is quite unusual in density estimation. Nevertheless it already appears in density deconvolution in a slightly different way in Pensky and Vidakovic (1999) who assume, instead of $\left(\mathrm{A}_{3}^{X}\right)$ that $\sup _{x \in \mathbb{R}}|x| g(x)<\infty$. It is important to note that Assumption $\left(\mathrm{A}_{3}^{X}\right)$ is very unrestrictive.

All densities having tails of order $|x|^{-(m+1)}$ as $x$ tends to infinity satisfy $\left(\mathrm{A}_{3}^{X}\right)$ only if $m>1 / 2$. One can cite for instance the Cauchy distribution or all stable distributions with exponent $r>1 / 2$ (see Devroye (1986)). But, the Levy distribution, with exponent $r=1 / 2$ does not satisfies $\left(\mathrm{A}_{3}^{X}\right)$.

\subsection{The projection spaces}

Consider $\varphi(x)=\sin (\pi x) /(\pi x)$, and let $\varphi_{m, j}(x)=\sqrt{L_{m}} \varphi\left(L_{m} x-j\right), m \in \mathcal{M}_{n}=\left\{1, \cdots, m_{n}\right\}$. It is well known (see for instance Meyer (1990), p.22) that $\left\{\varphi_{m, j}\right\}_{j \in \mathbb{Z}}$ is an orthonormal basis of the space of square integrable functions having a Fourier transform with compact support included 
into $\left[-\pi L_{m}, \pi L_{m}\right]$. We denote by $S_{m}$ such a space and by $\left(S_{m}\right)_{m \in \mathcal{M}_{n}}$ this collection of linear spaces. In other words

$$
S_{m}=\left\{\sum_{j \in \mathbb{Z}} a_{m, j} \varphi_{m, j}, a_{m, j} \in \mathbb{R}\right\}=\left\{f \in \mathbb{L}_{2}(\mathbb{R}), \text { with } \operatorname{supp}\left(f^{*}\right) \text { included into }\left[-L_{m} \pi, L_{m} \pi\right]\right\} .
$$

When $L_{m}=2^{m}$, the basis $\left\{\varphi_{m, j}\right\}$ is known as the Shannon basis, but we consider here that $L_{m}=m$.

In this context, since $g_{m}=\sum_{j \in \mathbb{Z}} a_{m, j} \varphi_{m, j}$ with $a_{m, j}=\left\langle g, \varphi_{m, j}>\right.$, the orthogonal projection of $g$ on $S_{m}$, involves infinite sums, we also consider the truncated spaces $S_{m}^{(n)}$ defined as

$$
S_{m}^{(n)}=\left\{\sum_{|j| \leq K_{n}} a_{m, j} \varphi_{m, j}, a_{m, j} \in \mathbb{R}\right\} \text { where } K_{n} \text { is an integer. }
$$

It is easy to see that, $\left\{\varphi_{m, j}\right\}_{|j| \leq K_{n}}$ is an orthonormal basis of $S_{m}^{(n)}$ and the orthogonal projection $g_{m}^{(n)}$ of $g$ on $S_{m}^{(n)}$ is given by $g_{m}^{(n)}=\sum_{|j| \leq K_{n}} a_{m, j} \varphi_{m, j}$ with $a_{m, j}=<g, \varphi_{m, j}>$.

Associate this collection of models to the following contrast function, for $t$ belonging to $S_{m}^{(n)}$

$$
\gamma_{n}(t)=\frac{1}{n} \sum_{i=1}^{n}\left[\|t\|^{2}-2 u_{t}^{*}\left(Z_{i}\right)\right], \quad \text { with } \quad u_{t}(x)=\frac{1}{2 \pi}\left(\frac{t^{*}(-x)}{f_{\varepsilon}^{*}(\sigma x)}\right) .
$$

By using Parseval and inverse Fourier formulas we get that

$$
\mathbb{E}\left[u_{t}^{*}\left(Z_{i}\right)\right]=\frac{1}{2 \pi}\left\langle\left(\frac{t^{*}(-.)}{f_{\varepsilon}^{*}(\sigma .)}\right)^{*}, g * f_{\varepsilon}\right\rangle=\frac{1}{2 \pi}\left\langle\frac{t^{*}(.)}{f_{\varepsilon}^{*}(-\sigma .)}, g^{*} f_{\varepsilon}^{*}(\sigma .)\right\rangle=\frac{1}{2 \pi}\left\langle t^{*}, g^{*}\right\rangle=\langle t, g\rangle,
$$

and hence $\mathbb{E}\left(\gamma_{n}(t)\right)=\|t-g\|^{2}-\|g\|^{2}$ which is minimal when $t=g$. This shows that $\gamma_{n}(t)$ suits well for the estimation of $g$.

\subsection{Construction of the minimum contrast estimators}

Associated to the collection of models, the collection of non-penalized estimators $\hat{g}_{m}^{(n)}$ of $g$ is defined by

$$
\hat{g}_{m}^{(n)}=\arg \min _{t \in S_{m}^{(n)}} \gamma_{n}(t) .
$$

By using that, $t \mapsto u_{t}$ is linear, and that $\left\{\varphi_{m, j}\right\}_{|j| \leq K_{n}}$ is an orthonormal basis of $S_{m}^{(n)}$, we have $\hat{g}_{m}^{(n)}=\sum_{|j| \leq K_{n}} \hat{a}_{m, j} \varphi_{m, j} \quad$ where $\quad \hat{a}_{m, j}=n^{-1} \sum_{i=1}^{n} u_{\varphi_{m, j}}^{*}\left(Z_{i}\right)$ and $\mathbb{E}\left(\hat{a}_{m, j}\right)=<g, \varphi_{m, j}>=a_{m, j}$.

\subsection{Construction of the minimum penalized contrast estimator}

We aim at finding the best model $\hat{m}$ in $\mathcal{M}_{n}$, based on the data and not on prior knowledge on the smoothness of $g$, such that the risk of the resulting estimator is almost as good as the risk of the best estimator in the family. The model selection is performed in an automatic way, using the following penalized criteria

$$
\tilde{g}=\hat{g}_{\hat{m}}^{(n)} \text { with } \hat{m}=\arg \min _{m \in \mathcal{M}_{n}}\left[\gamma_{n}\left(\hat{g}_{m}^{(n)}\right)+\operatorname{pen}(m)\right],
$$

where the penalty function $\operatorname{pen}(m)$ is defined by

$$
\operatorname{pen}(m)=2 a\left(\lambda_{1}+\mu \sigma^{\delta} \pi^{\delta} \lambda_{2}\right) \frac{L_{m}^{\max (0, \min (3 \delta / 2-1 / 2, \delta))} \Gamma(m)}{n},
$$


the constant $a$ is a fixed universal constant (to be found by simulation experiments),

$$
\begin{gathered}
\lambda_{1}\left(\gamma, \kappa_{0}, \mu, \sigma, \delta\right)=\frac{\left(\sigma^{2} \pi^{2}+1\right)^{\gamma}}{\pi^{\delta} \kappa_{0}^{2} R(\mu, \delta, \sigma)}, R(\mu, \delta, \sigma)=\mathbb{I}_{\{\delta=0\}}+2 \mu \delta \sigma^{\delta} \mathbb{I}_{\{0<\delta \leq 1\}}+2 \mu \sigma^{\delta} \mathbb{I}_{\{\delta>1\}}, \\
\lambda_{2}=\lambda_{1}^{1 / 2}\left(1+\sigma^{2} \pi^{2}\right)^{\gamma / 2}\left\|f_{\varepsilon}\right\| \kappa_{0}^{-1}(2 \pi)^{-1 / 2} \mathbb{I}_{\{1 / 3 \leq \delta \leq 1\}}+\lambda_{1} \mathbb{I}_{\{\delta>1\}}, \\
\text { and } \quad \Gamma(m)=L_{m}^{(2 \gamma+1-\delta)} \exp \left\{2 \mu \sigma^{\delta} \pi^{\delta} L_{m}^{\delta}\right\} .
\end{gathered}
$$

Since $\sigma$ and $f_{\varepsilon}$ are known, the constants $\sigma$ and $\mu, \delta, \kappa_{0}, \gamma$ defined in $\left(\mathrm{A}_{2}^{\varepsilon}\right)$ are also known.

\section{RATES OF CONVERGENCE OF THE MINIMUM CONTRAST ESTIMATORS $\hat{g}_{m}^{(n)}$}

\subsection{Bias-variance decomposition of risk of $\hat{g}_{m}^{(n)}$}

Let us first study the rate of convergence of one estimator $\hat{g}_{m}^{(n)}$, when the smoothness of $g$ is known.

Proposition 1. Under Assumption $\left(\mathrm{A}_{3}^{X}\right)$, denote by $\Delta_{1}(m)=L_{m} \int_{-\pi}^{\pi}\left|f_{\varepsilon}^{*}\left(L_{m} x \sigma\right)\right|^{-2} d x /(2 \pi)$. Then $\mathbb{E}\left\|g-\hat{g}_{m}^{(n)}\right\|^{2} \leq\left\|g-g_{m}\right\|^{2}+\left(\pi L_{m}\right)^{2}\left(M_{2}+1\right) / K_{n}+2 \Delta_{1}(m) / n$.

REMARK 1. We point out that the $\left\{\varphi_{m, j}\right\}$ are $\mathbb{R}$-supported (and not compactly supported) so that we obtain an estimation on $\mathbb{R}$ and not only on a compact set as for usual projection estimators. This is a great advantage of this basis. Nevertheless it induces the residual term $\left(\pi L_{m}\right)^{2}\left(M_{2}+1\right) / K_{n}$, due to the truncation $|j| \leq K_{n}$. But the most important thing is that the choice of $K_{n}$ does not influence the other terms. Consequently, it is easy to check that we can find a relevant choice of $K_{n}\left(K_{n} \geq n\right.$ under $\left(\mathrm{A}_{3}^{X}\right)$, that makes this last supplementary term unconditionally negligible with respect to the others. The choice of large $K_{n}$ does not change the efficiency of our estimator from a statistical point of view but only changes some practical computations.

Let us comment the three terms in the bound of the risk. The variance term $\Delta_{1}(m) / n$ depends on the rate of decay of the Fourier transform of $f_{\varepsilon}$, with larger variance for smoother $f_{\varepsilon}$. Under $\left(\mathrm{A}_{2}^{\varepsilon}\right)$, by applying Lemma 3 in Section 6.3 , we get that $\Delta_{1}(m) \leq 2 \lambda_{1} \Gamma(m)$ where $\Gamma(m)$ is given by (5) and $\lambda_{1}=\lambda_{1}\left(\gamma, \kappa_{0}, \mu, \sigma, \delta\right)$ is given by (4). In order to ensure that $\Gamma\left(m_{n}\right) / n$ is bounded, we only consider $L_{m}=m \leq m_{n}$ with

$$
m_{n} \leq \begin{cases}\pi^{-1} n^{1 /(2 \gamma+1)} & \text { if } \delta=0 \\ \pi^{-1}\left[\frac{\ln (n)}{2 \mu \sigma^{\delta}}+\frac{2 \gamma+1-\delta}{2 \delta \mu \sigma^{\delta}} \ln \left(\frac{\ln (n)}{2 \mu \sigma^{\delta}}\right)\right]^{1 / \delta} & \text { if } \delta>0 .\end{cases}
$$

$\operatorname{Under}\left(\mathrm{A}_{3}^{X}\right)$ and $\left(\mathrm{A}_{2}^{\varepsilon}\right)$, if $K_{n} \geq n$, then we have

$$
\mathbb{E}\left\|g-\hat{g}_{m}^{(n)}\right\|^{2} \leq\left\|g-g_{m}\right\|^{2}+2 \lambda_{1} \Gamma(m) / n+\left(\pi L_{m}\right)^{2}\left(M_{2}+1\right) / n
$$

Finally, since $g_{m}$ is the orthogonal projection of $g$ on $S_{m}$, we get that $g_{m}^{*}=g^{*} \mathbb{I}_{\left[-L_{m} \pi, L_{m} \pi\right]}$ and therefore $\left\|g-g_{m}\right\|^{2}=(2 \pi)^{-1}\left\|g^{*}-g_{m}^{*}\right\|^{2}=(2 \pi)^{-1} \int_{|x| \geq \pi L_{m}}\left|g^{*}\right|^{2}(x) d x$.

3.2 Order of the risk of $\hat{g}_{m}^{(n)}$ under regularity assumptions on $g$

Under $\left(\mathrm{R}_{2}^{X}\right)$ and $\left(\mathrm{A}_{3}^{X}\right)$, by choosing $\pi L_{m}=d$, and $K_{n} \geq n$, the bias term $\left\|g-g_{m}\right\|^{2}=0$, the bound $(7)$ becomes $\mathbb{E}\left(\left\|g-\hat{g}_{m}^{(n)}\right\|^{2}\right) \leq 2 \lambda_{1} d^{(2 \gamma+1-\delta)} \exp \left\{2 \mu \sigma^{\delta} \pi^{\delta} d^{\delta}\right\} / n+d^{2}\left(M_{2}+1\right) /\left(\pi^{2} n\right)$, and the density $g$ is estimated with the parametric rate of convergence. We refer to Ibragimov and 
Hasminskii (1983) for similar result on the "direct" estimation of a density $g$ satisfying Assumption $\left(\mathrm{R}_{2}^{X}\right)$, using the observations $X_{1}, \cdots, X_{n}$.

If now $g$ satisfies $\left(\mathrm{R}_{1}^{X}\right),\left\|g-g_{m}\right\|^{2} \leq\left[C_{1} /(2 \pi)\right]\left(L_{m}^{2} \pi^{2}+1\right)^{-s} \exp \left\{-2 b \pi^{r} L_{m}^{r}\right\}$. According to (7), under $\left(\mathrm{A}_{3}^{X}\right)$ with $K_{n} \geq n$, the risk of $\hat{g}_{m}^{(n)}$ is bounded by

$C_{1}(2 \pi)^{-1}\left(L_{m}^{2} \pi^{2}+1\right)^{-s} \exp \left\{-2 b \pi^{r} L_{m}^{r}\right\}+2 \lambda_{1} L_{m}^{(2 \gamma+1-\delta)} \exp \left\{2 \mu \sigma^{\delta} \pi^{\delta} L_{m}^{\delta}\right\} / n+\left(\pi L_{m}\right)^{2}\left(M_{2}+1\right) / n$.

The optimal choices of $L_{m}$ and the resulting rates are given in Table 1, for different types of smoothness of the unknown density $g$ and different types of known error density $f_{\varepsilon}$.

Table 1: Optimal choice of the length $\left(L_{\breve{m}}\right)$ and resulting (optimal) rates under Assumptions $\left(\mathrm{A}_{2}^{\varepsilon}\right)$ and $\left(\mathrm{R}_{1}^{X}\right)$.

\begin{tabular}{|c|c|c|c|}
\hline & \\
\hline & & $\begin{array}{c}\delta=0 \\
\text { ordinary smooth }\end{array}$ & $\begin{array}{c}\delta>0 \\
\text { supersmooth }\end{array}$ \\
\hline & $\begin{array}{l}r=0 \\
\operatorname{Sobolev}(s)\end{array}$ & $\begin{array}{l}\pi L_{\breve{m}}=O\left(n^{1 /(2 s+2 \gamma+1)}\right) \\
\text { rate }=O\left(n^{-2 s /(2 s+2 \gamma+1)}\right) \\
\text { minimax rate }\end{array}$ & $\begin{array}{l}\pi L_{\breve{m}}=\left[\ln (n) /\left(2 \mu \sigma^{\delta}+1\right)\right]^{1 / \delta} \\
\text { rate }=O\left((\ln (n))^{-2 s / \delta}\right) \\
\text { minimax rate }\end{array}$ \\
\hline$g$ & $\begin{array}{l}r>0 \\
\mathcal{C}^{\infty}\end{array}$ & $\begin{array}{l}\pi L_{\breve{m}}=[\ln (n) / 2 b]^{1 / r} \\
\text { rate }=O\left(\frac{\ln (n)^{(2 \gamma+1) / r}}{n}\right) \\
\text { minimax rate }\end{array}$ & 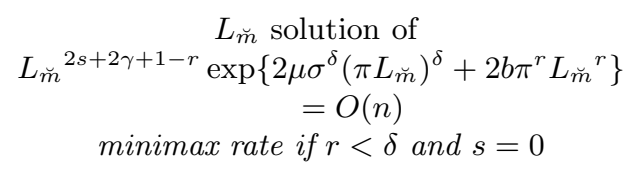 \\
\hline
\end{tabular}

Let us emphasize that the rate for $r>0, \delta>0$ is not explicitly given, but is only written the solution $L_{\breve{m}}$ of the equation

$$
L_{\breve{m}}^{2 s+2 \gamma+1-r} \exp \left\{2 \mu \sigma^{\delta}\left(\pi L_{\breve{m}}\right)^{\delta}+2 b \pi^{r} L_{\breve{m}}{ }^{r}\right\}=O(n) .
$$

The study of this case is of most importance since the case $\delta>0$ contains the most studied case of Gaussian errors. The association $\delta>0$ and $r=0$ leads usually people to conclude that this problem is without hope when $\delta>0$ since the rates, of logarithmic order, are indeed very slow in that case. But if we associate $\delta>0$ to $r>0$, then much faster than logarithmic rates are recovered (see Section 3.4). The empirical experiments of Section 5 illustrate that the estimation algorithm works well in that case. Lastly, we can mention that, in the context of stochastic volatility models seen as processes observed with errors, most stationary distributions of standard diffusion models studied by Comte and Genon-Catalot (2005) happen to belong to this class.

3.3 About the solution of Equation (8), in the case $r>0, \delta>0$

The special case $r=\delta>0$ leads to the explicit solution

$$
\pi L_{\breve{m}}=\left[\ln \left(n / \ln (n)^{a}\right) /\left(2 \mu \sigma^{\delta}+2 b\right)\right]^{1 / r} \text { with } a=(2 s+2 \gamma-r+1) / r
$$

and to the rate $[\ln (n)]^{a^{\prime}} n^{-a^{\prime} /\left(a^{\prime}+\mu \sigma^{\delta}\right)}$ with $a^{\prime}=\left(-2 s \mu \sigma^{\delta}+(2 \gamma-r+1) b\right) /\left(r\left(\mu \sigma^{\delta}+b\right)\right)$.

If $r>0, \delta>0$ and $r \neq \delta$, the expression of optimal parameter $L_{\breve{m}}$, solution of the Equation (8), has not one single form for general $r>0$ and $\delta>0$. 
- When $0<r<\delta$, we can precise here the order of the rate by using some additional information on the ratio $r / \delta<1$. We have to distinguish if $r / \delta \leq 1 / 2$ or $1 / 2<r / \delta \leq 2 / 3, \ldots$ More precisely, if $r / \delta \leq 1 / 2$, the optimal choice $L_{\breve{m}}$ is

$$
\pi L_{\breve{m}}=\left[\frac{\ln (n)}{2 \mu \sigma^{\delta}}-\frac{2 b}{2 \mu \sigma^{\delta}}\left(\frac{\ln (n)}{2 \mu \sigma^{\delta}}\right)^{r / \delta}-c \ln \left(\frac{\ln (n)}{2 \mu \sigma^{\delta}}\right)\right]^{1 / \delta} \quad \text { with } c=\frac{2 \gamma-r+2 s+1}{2 \mu \sigma^{\delta} \delta}
$$

and the rate is of order

$$
\ln (n)^{-2 s / \delta} \exp \left[-2 b\left(\ln (n) /\left(2 \mu \sigma^{\delta}\right)\right)^{r / \delta}\right] .
$$

If $1 / 2<r / \delta \leq 2 / 3$ the optimal choice of $\pi L_{\breve{m}}$ is

$$
\pi L_{\breve{m}}=\left[\frac{\ln (n)}{2 \mu \sigma^{\delta}}-\frac{2 b}{2 \mu \sigma^{\delta}}\left(\frac{\ln (n)}{2 \mu \sigma^{\delta}}\right)^{r / \delta}+\frac{r}{\delta} \frac{(2 b)^{2}}{2 \mu \sigma^{\delta}}\left(\frac{\ln (n)}{2 \mu \sigma^{\delta}}\right)^{2 r / \delta-1}-c \ln \left(\frac{\ln (n)}{2 \mu \sigma^{\delta}}\right)\right]^{1 / \delta}
$$

with the same $c$ as above, which gives the rate

$$
\ln (n)^{-2 s / \delta} \exp \left[-2 b\left(\frac{\ln (n)}{2 \mu \sigma^{\delta}}\right)^{r / \delta}+\frac{(2 b)^{2}}{2 \mu \sigma^{\delta}} \frac{r}{\delta}\left(\frac{\ln (n)}{2 \mu \sigma^{\delta}}\right)^{2 r / \delta-1}\right] .
$$

If $2 / 3<r / \delta \leq 3 / 4$, we have another choice of $\pi L_{\breve{m}}$ with another rate.

- When $0<\delta<r$, we can also precise the order of the rate of our estimator, by using, once again, some additional information on the ratio $\delta / r$. For instance, if $\delta / r \leq 1 / 2$, the optimal choice $L_{\breve{m}}$ is

$$
\pi L_{\breve{m}}=\left[\frac{\ln (n)}{2 b}-\frac{2 \mu \sigma^{\delta}}{2 b}\left(\frac{\ln (n)}{2 b}\right)^{\delta / r}-c \ln \left(\frac{\ln (n)}{2 b}\right)\right]^{1 / r} \quad \text { with } c=\frac{2 \gamma-r+2 s+1}{2 b r}
$$

and the rate is of order

$$
\ln (n)^{(2 \gamma+1-\delta) / r} \exp \left[2 \mu \sigma^{\delta}(\ln (n) /(2 b))^{\delta / r}\right] / n .
$$

As in the case $0<r<\delta$, we obtain a different rate for $1 / 2<\delta / r<2 / 3$.

It follows that in the case $r>0$ and $\delta>0$, the rate depends on the integer $k$ such that $r / \delta$ or $\delta / r$ belongs to the interval $\left.\left.I_{k}=\right] k /(k+1) ;(k+1) /(k+2)\right]$. We are, to our knowledge, the first ones to have noticed this (unavoidable) particularity of the rates.

3.4 About the optimality of $\hat{g}_{m}^{(n)}$ when $g$ belongs to $\mathcal{S}_{s, r, b}\left(C_{1}\right)$

The rates $n^{-2 s /(2 s+2 \gamma+1)}(\delta=0, r=0), \ln (n)^{-2 s / \delta}(\delta>0, r=0)$ and $\ln (n)^{(2 \gamma+1) / r} / n(\delta=0, r>0)$ are known to be the minimax rates and we refer to Fan (1991) (first two cases) and to Butucea (2004) (last case) for lower bounds.

The optimality of the rates in the case $\delta>0, r>0$ requires a specific discussion.

To our knowledge, the first paper dealing with the case where $g$ is super smooth $(r>0)$ is the paper by Pensky and Vidakovic (1999). See Section 4.3 for a discussion of the rates they obtain compared to ours.

The case $r=\delta=1$ is studied by Tsybakov (2000) and Cavalier et al. (2003), in the case of inverse problems with random noise. In this case and in both problems (density deconvolution and inverse problem) the best compromise is explicit and so is the rate of convergence, of order 
$n^{-a^{\prime} /\left(a^{\prime}+\mu \sigma\right)}[\ln n]^{(-2 s \mu \sigma+2 b \gamma) /(\mu \sigma+b)}$. It is noteworthy that $\hat{g}_{m}^{(n)}$ seems also to achieve the minimax rate of convergence in this case.

When $0<r<\delta$, some lower bounds are known in the special case $0<r<\delta$ and $s=0$. According to Butucea and Tsybakov (2004), in this case, if we denote by $\pi L_{\breve{m}}$ the solution of $2 \mu \sigma^{\delta}\left(\pi L_{\breve{m}}\right)^{\delta}+2 b\left(\pi L_{\breve{m}}\right)^{r}=\ln n-(\ln \ln n)^{2}$, then the rate of convergence of $\hat{g}_{m}$ is the minimax rate of order $\exp \left\{-2 b\left(\pi L_{\breve{m}}\right)^{r}\right\}$. The rate of convergence is always of order a power of $\ln (n)$ multiplied by an exponential term, that is decreases faster that any logarithmic function, but slower than any power of $n$.

When $0<\delta<r$, no lower bounds are available. In this case, the rate is of order a power of $\ln (n)$ multiplied by a negative power of $n$ and by an exponential term.

\subsection{Conclusion on the minimum contrast estimators $\hat{g}_{m}^{(n)}$}

The estimator $\hat{g}_{m}^{(n)}$ achieves the minimax rate in all cases where lower bounds are available but its construction requires the knowledge of the smoothness of $g$. All those facts give strong motivation to find some adaptive estimation procedure that does not require such prior smoothness knowledge on $g$, and whose risk automatically achieves the minimax rate.

\section{ADAPTIVE ESTIMATION}

\subsection{Main result of adaptive estimation}

We look for a penalty function, based on the observations and on $\sigma f_{\varepsilon}(\cdot / \sigma)$, such that, for $K_{n} \geq n$

$$
\mathbb{E}\|\tilde{g}-g\|^{2} \leq \inf _{m \in \mathcal{M}_{n}}\left[\left\|g-g_{m}\right\|^{2}+\left(\pi L_{m}\right)^{2}\left(M_{2}+1\right) / n+2 \lambda_{1} \Gamma(m) / n\right]
$$

The following theorem describes the cases where the oracle inequality (11) is reached.

THEOREM 1. Under the assumptions $\left(\mathrm{A}_{2}^{\varepsilon}\right)$ and $\left(\mathrm{A}_{3}^{X}\right)$, consider the collection of estimators $\hat{g}_{m}^{(n)}$ defined by (1) with $K_{n} \geq n$ and $1 \leq m \leq m_{n}$ satisfying (ब) if $\delta \leq 1 / 3$ and if $\delta>1 / 3$,

$$
m_{n} \leq \pi^{-1}\left[\frac{\ln (n)}{2 \mu \sigma^{\delta}}+\frac{2 \gamma+1-\delta+\min ((3 \delta / 2-1 / 2), \delta)}{2 \delta \mu \sigma^{\delta}} \ln \left(\frac{\ln (n)}{2 \mu \sigma^{\delta}}\right)\right]^{1 / \delta} .
$$

Let $\operatorname{pen}(m)$ be defined by (3) for some universal numerical constant $a>1$. Then, $\tilde{g}=\hat{g}_{\hat{m}}^{(n)}$ defined by (4) satisfies

$$
\mathbb{E}\left(\|g-\tilde{g}\|^{2}\right) \leq C_{a} \inf _{m \in\left\{1, \ldots, m_{n}\right\}}\left[\left\|g-g_{m}\right\|^{2}+\operatorname{pen}(m)+\left(\pi L_{m}\right)^{2}\left(M_{2}+1\right) / n\right]+a \kappa_{a} C / n,
$$

where $C_{a}=\max \left(\kappa_{a}^{2}, 2 \kappa_{a}\right), \kappa_{a}=(a+1) /(a-1)$ and $C$ is a constant depending on $f_{\varepsilon}$ and $\sigma$.

Obviously, Remark 1 still holds for the adaptive estimator.

The rates are easy to deduce from (12) as soon as $g$ belongs to some smoothness class, but the procedure will reach the rate without requiring the knowledge of any smoothness parameter.

\subsection{About the optimality of the adaptive estimator $\tilde{g}$}

Rate of $\tilde{g}$ under $\left(\mathrm{R}_{2}^{X}\right)$ : no loss. 
If $g$ satisfies $\left(\mathrm{R}_{2}^{X}\right)$, then according to Section $3.2,\left\|g-g_{m}\right\|^{2}=0$ as soon as $\pi L_{m} \geq d$, and the parametric rate of convergence is automatically achieved without the knowledge of $C_{2}$ and $d$ and especially without requiring to know that $\left(\mathrm{R}_{2}^{X}\right)$ is fulfilled.

Rate of $\tilde{g}$ under $\left(\mathrm{R}_{1}^{X}\right)$.

Under $\left(\mathrm{R}_{1}^{X}\right)$, the rate of convergence of $\tilde{g}$ clearly depends on the order of the penalty compared to the variance order $\Gamma(m) / n$. If $g$ satisfies $\left(\mathrm{R}_{1}^{X}\right),\left\|g-g_{m}\right\|^{2} \leq\left(C_{1} / 2 \pi\right) L_{m}^{-2 s} \exp \left\{-2 b \pi^{r} L_{m}^{r}\right\}$. For instance, if $\delta=0$, by associating the order of the bias to the value of $\operatorname{pen}(m)$, of order $\Gamma(m) / n$, we obtain that the estimator $\tilde{g}$ automatically reaches the minimax rate $\ln (n)^{(2 \gamma+1) / r} / n$, without the knowledge of $s, r$ nor $b$. In all cases, $\tilde{g}$ achieves the minimax rate up to some logarithmic factor.

Rate of $\tilde{g}$ under $\left(\mathrm{R}_{1}^{X}\right)$, cases without loss.

When $0 \leq \delta \leq 1 / 3$, the penalty function has the variance order $\Gamma(m) / n$, and $\tilde{g}$ achieves the best rate of $\hat{g}_{\breve{m}}$. Under $\left(\mathrm{R}_{1}^{X}\right)$, this best rate is the minimax rate in all cases here, except if $r \geq \delta>0$ and $\delta \leq 1 / 3$ which is a case where no lower bounds are available.

When $\delta>1 / 3$, the penalty function $\operatorname{pen}(m)$ has not exactly the order of the variance $\Gamma(m) / n$, but a loss of order $L_{m}^{\min ((3 \delta / 2-1 / 2), \delta)}$ occurs, that is of order $L_{m}^{(3 \delta-1) / 2}$ if $1 / 3<\delta \leq 1$ and of order $L_{m}^{\delta}$ if $\delta>1$. Consequently $\tilde{g}$ achieves the best rate of $\hat{g}_{\breve{m}}$ if the bias $\left\|g-g_{m}\right\|^{2}$ is the dominating term in the trade-off between $\left\|g-g_{m}\right\|^{2}$ and pen $(m)$.

- When $r=0$ and $\delta>1 / 3$, the minimax rate of order $(\ln (n))^{-2 s / \delta}$ is given by the bias term, and the loss in the penalty function does not change the rate achieved by the adaptive estimator $\tilde{g}$, which remains thus the minimax rate.

- When $0<r<\delta$, the rate is given by the bias term and thus this loss does not affect the rate of convergence of $\tilde{g}$ either. Therefore, $\tilde{g}$ achieves the best rate of $\hat{g}_{\breve{m}}$, which is the minimax rate of convergence when $s=0$ and also probably if $s \neq 0$. In the specific case $0<r<\delta / 2$ and $s=0$, Butucea and Tsybakov (2004) also propose an adaptive estimator. But this requires to know that $0<r<\delta / 2$ and $s=0$.

Rate of $\tilde{g}$ under $\left(\mathrm{R}_{1}^{X}\right)$, case with loss.

- When $r \geq \delta>1 / 3$, pen $(m)$ can be the dominating term in the trade-off between $\left\|g-g_{m}\right\|^{2}$ and pen $(m)$. This induces a loss of order $L_{m}^{\min ((3 \delta / 2-1 / 2), \delta)}$ in the rate of convergence of $\tilde{g}$ compared to the best rate of $\hat{g}_{\breve{m}}$. Since it happens in cases where the order of the optimal $L_{m}$ is less than $(\ln n)^{1 / \delta}$, the loss in the rate is at most of order $\ln n$, when the rate is faster than logarithmic and consequently, the loss appears only in cases where it can be seen as negligible.

For $\mathbb{L}_{2}$ estimation, such an unavoidable logarithmic loss in adaptation, has been pointed out by Tsybakov (2000) and Cavalier et al. (2003) in case of inverse problems with random noise, when $r=\delta=1$, which shows, in a slightly different model but with comparable rates of convergence, that a loss due to adaptivity of order $\ln (n)^{b /(\mu \sigma+b)}$ is unavoidable. The main point is that, according to (9), our estimator has its quadratic risk with the same logarithmic loss when $r=\delta=1$. This logarithmic loss due to adaptation seems thus unavoidable at least in one case.

REMARK 2. When $\sigma=0$, then by convention $\delta=\mu=0, \lambda_{1}=1$ and $\operatorname{pen}(m)=6 a L_{m} / n$ which is the penalty function used in direct density estimation. More precisely, if $\sigma$ is very small, then the procedure selects the parameter $L_{m}$ closed to the parameter selected in usual density estimation.

\subsection{Comparison with Pensky and Vidakovic (1999)}

To our knowledge, the first paper dealing with adaptive density deconvolution is the paper by Pensky and Vidakovic (1999) who are also the first that consider the case of $r>0$. The adaptive 
estimators proposed in Pensky and Vidakovic (1999) achieve minimax rates of convergence in the three cases $(\delta=0, r=0),(\delta=0, r>0)$, and $(\delta>0, r=0)$.

But when $(r>0, \delta>0)$, the rate of convergence of their estimator is not minimax. This is shown in the special case $0<r<\delta$ and $s=0$, in Butucea and Tsybakov (2004), where sharp minimax results are stated. This is also shown by our results when $0<\delta \leq r$ and when $0<r<\delta$, $s \neq 0$ (see Sections 3.4 and 4.2). For instance, when $0<\delta / r \leq 1 / 2$, according to (10) and Sections 3.3 and 4.2 , the resulting rate of $\tilde{g}$ is of order

$$
\ln (n)^{\max (0, \min (3 \delta / 2-1 / 2, \delta) / r)} \ln (n)^{(2 \gamma+1-\delta) / r} \exp \left[2 \mu \sigma^{\delta}(\ln (n) /(2 b))^{\delta / r}\right] / n
$$

stricly faster than the upper bound of the rate in Pensky and Vidakovic (1999) (see their Theorem 4) which is of order $\ln (n)^{(2 \gamma+1) / \delta} / n^{\Lambda /\left(\Lambda+2 \mu \sigma^{\delta}(4 \pi / 3)^{\delta}\right)}$ for $\Lambda>0$.

The non-optimality of their adaptive estimator when $(\delta>0, r>0)$ comes from two facts. First, when $(\delta>0, r>0)$, they choose a smoothing parameter (analogous to $L_{\breve{m}}$ ) as in the case $(r=0, \delta>0)$. Consequently, it provides an adaptive estimator in the sense that it does not depend on the smoothness parameters of $g$. But it does not give the best rate for their estimator, since it does not correspond to the best choice in their bias-variance compromise.

Second, this non optimality of their estimator when $\delta>0, r>0$, comes also, in a more crucial manner, from the fact that their wavelet and scaling functions cannot provide the optimal bias-variance decomposition. This is due to the support of the Fourier transform of their scaling function as well as their wavelet which induce, when $\delta>0, r>0$, a squared bias term of order $L_{m}^{-2 s} \exp \left\{-2 b(2 \pi / 3)^{r} L_{m}^{r}\right\}$ with a variance term of order $L_{m}^{2 \gamma+1-\delta} \exp \left\{2 \mu \sigma^{\delta}(4 \pi / 3)^{\delta} L_{m}^{\delta}\right\}$. When either $(\delta=0, r=0),(\delta>0, r>0)$ or $(\delta>0, r=0)$, those supports have no influence on the rate of convergence, and hence their estimator is minimax. But these supports do not allow to reach the minimax rate when $(\delta>0, r>0)$.

The asymptotic properties of $\tilde{g}$ are improved by using the basis generated by $\sin (\pi x) /(\pi x)$. Indeed, due to its Fourier transform, it implies a squared bias of order $L_{m}^{-2 s} \exp \left\{-2 b \pi^{r} L_{m}^{r}\right\}$ and a variance of order $L_{m}^{2 \gamma+1-\delta} \exp \left\{2 \mu \sigma^{\delta} \pi^{\delta} L_{m}^{\delta}\right\}$ and hence a better trade-off between the two terms. Section 3.3 as well as Butucea and Tsybakov (2004)'s results illustrate that the best choice of $L_{\breve{m}}$, solution of the bias-variance compromise (see equation (8)), requires quite precise computations. Besides its simplicity, this basis seems thus the most relevant since it gives the minimax rates in all the cases where lower bounds are available and faster rates than the ones in Pensky and Vidakovic (1999) in the remainder case.

\section{SIMULATION STUDY}

The implementation is conducted by using Matlab software. Details about the algorithm can obtained from the authors upon request. We choose $K_{n}=2^{8}$ as being of order $O(n)$ is all cases.

The integrated squared error $\operatorname{ISE}\left(\hat{g}_{\hat{m}}^{(n)}\right)=\left\|\hat{g}_{\hat{m}}^{(n)}-g\right\|^{2}$ is computed via a standard approximation and discretization of the integral on an interval of $\mathbb{R}$ denoted by $I$ and given in each case.

Then the $\operatorname{MISE}, \operatorname{MiSE}\left(\hat{g}_{\hat{m}}^{(n)}\right)=\mathbb{E}\left\|\hat{g}_{\hat{m}}^{(n)}-g\right\|^{2}$ is computed as the empirical mean of the approximated ISE $\left\|\hat{g}_{m}^{(n)}-g\right\|^{2}$, over 500 simulation samples. We illustrate our method on some test densities, with various smoothness properties, and for the two types of errors, ordinary and super smooth. We start by describing the error densities and the associated penalties.

\subsection{Two settings for the errors and the associated penalties}

We consider two types of error density $f_{\varepsilon}$, the first one is ordinary smooth, with polynomial decay of the Fourier Transform, and the second one is supersmooth, with an exponential decay of the Fourier transform $f_{\varepsilon}^{*}$. 
- Case 1: Laplace (or Double exponential) $\varepsilon$ 's. In this case, $f_{\varepsilon}(x)=e^{-\sqrt{2}|x|} / \sqrt{2}$, and $f_{\varepsilon}^{*}(x)=\left(1+x^{2} / 2\right)^{-1}$.

This density satisfies $\left(\mathrm{A}_{2}^{\varepsilon}\right)$ with $\gamma=2, \kappa_{0}=1 / 2$ and $\mu=\delta=0$.

According to Theorem 1, the penalty function, as the variance, is of order

$$
\frac{L_{m}}{n} \int_{-\pi}^{\pi}\left|\frac{\varphi^{*}(x)}{f_{\varepsilon}^{*}\left(\sigma L_{m} x\right)}\right|^{2} d x, \text { where } \int_{-\pi}^{\pi}\left|\frac{\varphi^{*}(x)}{f_{\varepsilon}^{*}\left(\sigma L_{m} x\right)}\right|^{2} d x=2 \pi\left(1+\frac{\pi^{2}}{3} \sigma^{2} L_{m}^{2}+\frac{\pi^{4}}{20} \sigma^{4} L_{m}{ }^{4}\right) .
$$

Some intensive simulation studies on various tested densities lead to choose the following penalty

$$
\operatorname{pen}\left(L_{m}\right)=\frac{6 \pi L_{m}}{n}\left(1+\frac{\left(\ln \left(L_{m}\right)\right)^{2.5}}{L_{m}}+\frac{\pi^{2}}{3} \sigma^{2} L_{m}{ }^{2}+\frac{\pi^{4}}{20} \sigma^{4} L_{m}{ }^{4}\right)
$$

- Case 2: Gaussian $\varepsilon$ 's. In that case, $f_{\varepsilon}(x)=1 / \sqrt{2 \pi} e^{-x^{2} / 2}$, and $f_{\varepsilon}^{*}(x)=e^{-x^{2} / 2}$.

This density satisfies $\left(\mathrm{A}_{2}^{\varepsilon}\right)$ with $\gamma=0, \kappa_{0}=1, \delta=2$ and $\mu=1 / 2$.

According to Theorem 1, the penalty, slightly bigger than the variance term, is of order

$$
\frac{L_{m}^{3}}{n} \int_{-\pi}^{\pi}\left|\frac{\varphi^{*}(x)}{f_{\varepsilon}^{*}\left(\sigma L_{m} x\right)}\right|^{2} d x \quad \text { where } \quad \int_{-\pi}^{\pi}\left|\frac{\varphi^{*}(x)}{f_{\varepsilon}\left(\sigma L_{m} x\right)}\right|^{2} d x=\int_{-\pi}^{\pi} \exp \left(\sigma^{2} L_{m}{ }^{2} x^{2}\right) d x .
$$

As in the previous case, some intensive simulation studies on various tested densities lead to choose the following penalty

$$
\operatorname{pen}\left(L_{m}\right)=\frac{6 \pi L_{m}}{n}\left(1+\frac{\left(\ln \left(L_{m}\right)\right)^{2.5}}{L_{m}}+\frac{\pi^{2} \sigma^{2} L_{m}^{2}}{3}\right)\left(\int_{0}^{\pi} \exp \left(\sigma^{2} L_{m}^{2} x^{2}\right) d x / \pi\right),
$$

where the integral is numerically computed. According to the theory (see Theorem 1 , the loss due to the adaptation is the term $\pi^{2} \sigma^{2} L_{m}^{2} / 3$.

The additional term $\left(\ln \left(L_{m}\right)\right)^{2.5} / L_{m}$ is motivated by the works of Birgé and Rozenholc (2005). In our case also, this term improves the quality of the results by making the penalties slightly heavier when $L_{m}$ becomes large.

Note that when $\sigma=0$, both penalties are equal to $\left(6 \pi L_{m}\right)\left(1+\left(\ln \left(L_{m}\right)\right)^{2.5} / L_{m}\right) / n$.

\subsection{Test densities}

First we consider densities having classical smoothness properties like Hölderian smoothness with polynomial decay of their Fourier transform. Second we consider densities having stronger smoothness properties, with exponential decay of the Fourier transform. Except in the case of the infinite variance density (Cauchy density), we consider density functions $g$ normalized with unit variance so that $1 / \sigma^{2}$ represents the usual signal-to-noise ratio (variance of the signal divided by the variance of the noise) and is denoted in the sequel by $s 2 n$ defined as $s 2 n=1 / \sigma^{2}$. The functions which are considered are listed below, associated with the interval $I$ used to evaluate the ISE:

(a) Chi2(3)-type distribution, $X=1 / \sqrt{6} U, g_{X}(x)=\sqrt{6} g(\sqrt{6} x), U \sim \chi^{2}(3)$ where we know that $U \sim \Gamma\left(\frac{3}{2}, \frac{1}{2}\right)$,

and $I=[-1,16]$.

(b) Laplace distribution, $I=[-5,5]$.

(c) Mixed Gamma distribution, $X=1 / \sqrt{5.48} W$ with $W \sim 0.4 \Gamma(5,1)+0.6 \Gamma(13,1)$, and $I=[-1.5,26]$.

(d) Cauchy distribution, $g(x)=(1 / \pi)\left(1 /\left(1+x^{2}\right)\right), g^{*}(x)=e^{-|x|}, I=[-10,10]$.

(e) Gaussian distribution, $X \sim \mathcal{N}\left(0, \sigma^{2}\right)$ with $\sigma=1, I=[-4,4]$.

(f) Mixed Gaussian distribution: $X \sim \sqrt{2} V$ with $V \sim 0.5 \mathcal{N}(-3,1)+0.5 \mathcal{N}(2,1)$ and $I=[-8,7]$. 

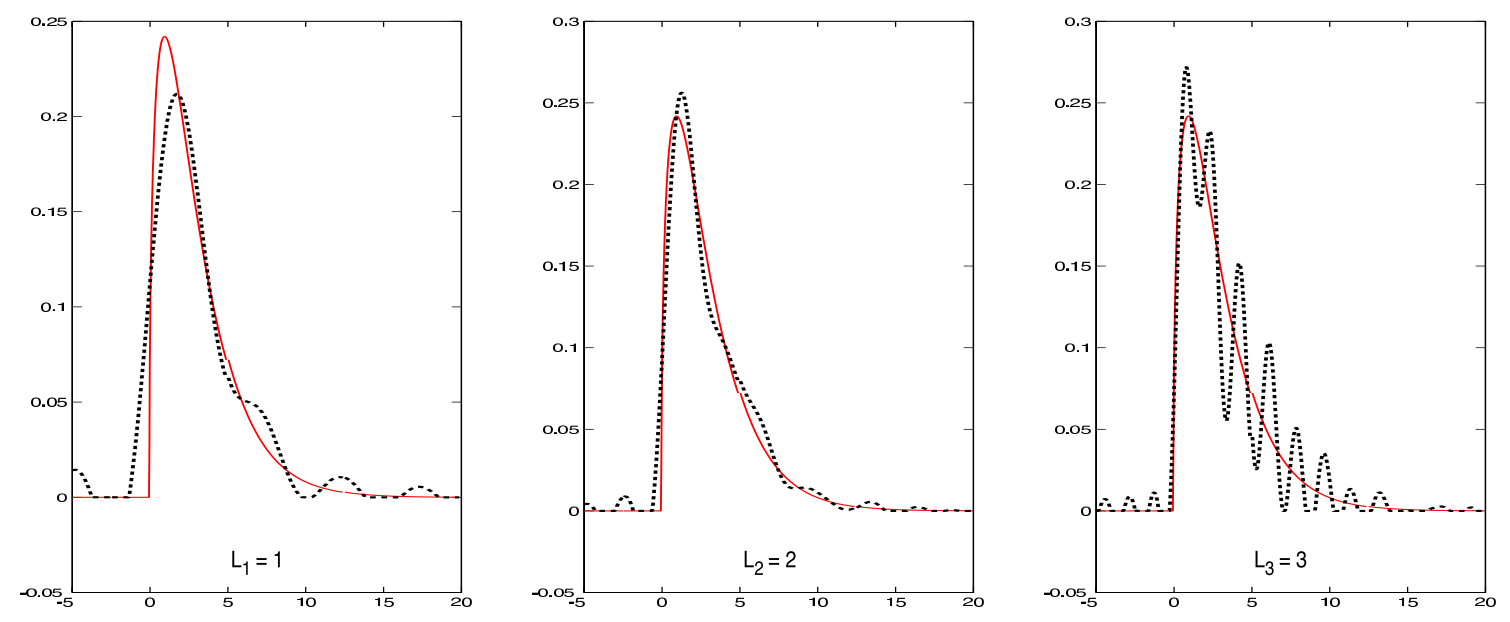

Figure 1: Plots of the estimator (dotted line) and of the true $\chi^{2}(3)$ (a) density (full line) - Laplace errors $-n=750, s 2 n=10$, when $L_{1}=1$ (left), $L_{2}=2$ (middle), $L_{3}=3$ (right). The algorithm chooses $\hat{m}=L_{\hat{m}}=2$.

Densities (a), (b), (c) correspond to cases with $r=0$, whereas densities (d), (e), (f) correspond to cases with $r>0$.

\subsection{Results}

Figure 1 compares the estimators $\hat{g}_{m}^{(n)}$ obtained for $m=L_{m}=1,2$ and 3 , and justifies the good choice $\hat{m}=2$ of the algorithm. Table 2 presents the MISE for the two types of errors, the different tested densities, different $s 2 n$ and for different sample sizes. The greatest values of $s 2 n$ amount to consider that there is essentially no noise. Clearly the MISE are smaller when there is less noise ( $\sigma$ small, $s 2 n$ large).

We can in particular compare the performances of our adaptive estimator with the performances of the deconvolution kernel as presented in Delaigle and Gijbels (2004a). This comparison is done for densities (a), (c), (e) and (f) which correspond to the densities \#2, \#6, \#1 and \#3 respectively, in Delaigle and Gijbels (2004a). They give median ISE obtained with kernel estimators by using four different methods of bandwidth selection. The comparison is given in Table 3 between the median ISE computed for 500 samples generated with the same interval length and signal to noise ratio as Delaigle and Gijbels (2004a). The ISE are computed on the same intervals $I$ as them. We also give our corresponding means since we believe that they are more meaningful than medians since the MISE is $\mathbb{E}\left\|\hat{g}_{m}^{(n)}-g\right\|^{2}$, but we also give our medians.

We can see that our estimation procedure provides better results in all cases except in one case, namely when we aim at estimating a Gaussian density, for both types of errors density. This is the most probably due to the fact that the bandwidth selection methods are based on computations assuming that the underlying density is Gaussian, so that they perform very well when it is true. For the other cases, even our means are often better than Delaigle and Gijbels'(2004a) medians which shows that our method provides a very good solution to the deconvolution problem.

A standard objection to deconvolution methods is that they require the knowledge of the noise density. Therefore, following the ideas of Meister (2004), we study here the properties of the estimator when the error density is not correctly specified. For both type of errors, we study the behavior of the estimator using one type of the error density when the other type of errors density is the good one. Table 4 presents the ratio between the resulting MISE if the errors density is 
Table 2: Mean MISE $\times 100$ obtained with $N=500$ samples, for sample sizes $n=$ $100,250,500,1000,2500$ and $s 2 n=2,4,10,100,1000$, the higher $s 2 n$ the lower the noise level. Densities (a): Chi2(3), (b): Laplace, (c): Mixed Gamma, (d): Cauchy, (e) Gaussian, (f): Mixed Gaussian.

\begin{tabular}{|c|c|c|c|c|c|c|c|c|c|c|c|}
\hline \multicolumn{2}{|c|}{$\times 10^{-2}$} & \multicolumn{2}{|c|}{$n=100$} & \multicolumn{2}{|c|}{$n=250$} & \multicolumn{2}{|c|}{$n=500$} & \multicolumn{2}{|c|}{$n=1000$} & \multicolumn{2}{|c|}{$n=2500$} \\
\hline$g$ & $s 2 n$ & Lap. & Gaus. & Lap. & Gaus. & Lap. & Gaus. & Lap. & Gaus. & Lap. & Gaus. \\
\hline \multirow{5}{*}{ (a) } & 2 & 2.02 & 4.15 & 1.39 & 2.37 & 1.18 & 1.72 & 1.06 & 1.36 & 1.03 & 1.12 \\
\hline & 4 & 1.52 & 1.79 & 1.21 & 1.27 & 1.07 & 1.13 & 1.04 & 1.04 & 0.654 & 0.996 \\
\hline & 10 & 1.31 & 1.31 & 1.13 & 1.11 & 1.01 & 1.03 & 0.505 & 0.995 & 0.345 & 0.974 \\
\hline & $10^{2}$ & 1.22 & 1.23 & 0.72 & 0.884 & 0.409 & 0.411 & 0.327 & 0.335 & 0.179 & 0.232 \\
\hline & $10^{3}$ & 1.22 & 1.21 & 0.651 & 0.638 & 0.391 & 0.382 & 0.293 & 0.298 & 0.157 & 0.157 \\
\hline \multirow{5}{*}{ (b) } & 2 & 3.7 & 10.6 & 2.17 & 5.2 & 1.61 & 3.03 & 1.41 & 2.07 & 1.2 & 1.48 \\
\hline & 4 & 2.5 & 2.99 & 1.66 & 1.93 & 1.33 & 1.46 & 1.26 & 1.25 & 0.817 & 1.12 \\
\hline & 10 & 1.9 & 1.97 & 1.43 & 1.42 & 1.35 & 1.22 & 0.723 & 1.12 & 0.441 & 1.06 \\
\hline & $10^{2}$ & 1.69 & 1.64 & 0.883 & 1.06 & 0.607 & 0.538 & 0.453 & 0.385 & 0.343 & 0.211 \\
\hline & $10^{3}$ & 1.68 & 1.65 & 0.814 & 0.79 & 0.593 & 0.561 & 0.411 & 0.379 & 0.284 & 0.24 \\
\hline \multirow{5}{*}{ (c) } & 2 & 1.32 & 3.96 & 0.547 & 1.88 & 0.292 & 1.01 & 0.148 & 0.533 & 0.06 & 0.224 \\
\hline & 4 & 0.79 & 1.05 & 0.316 & 0.453 & 0.151 & 0.224 & 0.0815 & 0.116 & 0.0361 & 0.0497 \\
\hline & 10 & 0.495 & 0.524 & 0.194 & 0.215 & 0.103 & 0.11 & 0.0543 & 0.0565 & 0.024 & 0.0246 \\
\hline & $10^{2}$ & 0.369 & 0.384 & 0.152 & 0.149 & 0.0789 & 0.0785 & 0.0409 & 0.0412 & 0.0194 & 0.0186 \\
\hline & $10^{3}$ & 0.364 & 0.353 & 0.149 & 0.15 & 0.0762 & 0.0767 & 0.0404 & 0.0406 & 0.0184 & 0.0185 \\
\hline \multirow{5}{*}{ (d) } & 2 & 2.72 & 9.09 & 1.22 & 4.26 & 0.645 & 2.3 & 0.353 & 1.25 & 0.158 & 0.513 \\
\hline & 4 & 1.66 & 2.27 & 0.716 & 0.967 & 0.364 & 0.514 & 0.205 & 0.28 & 0.138 & 0.127 \\
\hline & 10 & 1.15 & 1.13 & 0.437 & 0.46 & 0.249 & 0.257 & 0.215 & 0.142 & 0.219 & 0.0764 \\
\hline & $10^{2}$ & 0.815 & 0.783 & 0.373 & 0.351 & 0.351 & 0.271 & 0.206 & 0.201 & 0.147 & 0.0962 \\
\hline & $10^{3}$ & 0.783 & 0.78 & 0.366 & 0.355 & 0.34 & 0.331 & 0.189 & 0.189 & 0.121 & 0.118 \\
\hline \multirow{5}{*}{ (e) } & 2 & 2.74 & 9.21 & 1.1 & 4.08 & 0.605 & 2.14 & 0.296 & 1.06 & 0.143 & 0.446 \\
\hline & 4 & 1.59 & 2.23 & 0.591 & 0.878 & 0.362 & 0.457 & 0.229 & 0.227 & 0.463 & 0.0894 \\
\hline & 10 & 0.885 & 1.02 & 0.397 & 0.42 & 0.372 & 0.21 & 0.515 & 0.112 & 0.229 & 0.046 \\
\hline & $10^{2}$ & 0.711 & 0.713 & 0.565 & 0.432 & 0.396 & 0.394 & 0.279 & 0.195 & 0.171 & 0.15 \\
\hline & $10^{3}$ & 0.739 & 0.705 & 0.606 & 0.592 & 0.352 & 0.355 & 0.259 & 0.246 & 0.167 & 0.145 \\
\hline \multirow{5}{*}{ (f) } & 2 & 2.97 & 9.98 & 1.26 & 4.45 & 0.693 & 2.31 & 0.328 & 1.26 & 0.132 & 0.509 \\
\hline & 4 & 1.73 & 2.37 & 0.709 & 1.02 & 0.375 & 0.478 & 0.185 & 0.257 & 0.0751 & 0.105 \\
\hline & 10 & 1.14 & 1.21 & 0.463 & 0.466 & 0.237 & 0.242 & 0.118 & 0.122 & 0.0468 & 0.0515 \\
\hline & $10^{2}$ & 0.851 & 0.817 & 0.359 & 0.352 & 0.166 & 0.167 & 0.0866 & 0.0867 & 0.034 & 0.0351 \\
\hline & $10^{3}$ & 0.823 & 0.828 & 0.344 & 0.327 & 0.169 & 0.163 & 0.0845 & 0.0839 & 0.0334 & 0.0336 \\
\hline
\end{tabular}


not correct with the MISE if the errors density is correct. For instance, in the columns " $\varepsilon$ Lap." the noise density is Laplace but the MISE in the numerator of the ratio corresponds to estimators constructed as if it were Gaussian. As expected, since the construction uses the knowledge of the error density, if it is misspecified, the estimator presents some bias and the MISE becomes slightly bigger. Nevertheless, this difference does not clearly appear when $n$ is not very large. Indeed in that case, the optimal length $L_{m}$ is small and therefore the variance term of order $\int_{0}^{\pi L_{m}}\left|f_{\varepsilon}^{*}(x)\right|^{-2} d x$ is not so different between the two errors.

Table 3: Median ISE obtained by Delaigle and Gijbels (2004a) with a kernel estimator and four different strategies of bandwidth selection, and with our penalized projection estimator (median and mean).

\begin{tabular}{clcccc}
\hline & & \multicolumn{2}{c}{$n=100$} & \multicolumn{2}{c}{$n=250$} \\
\hline density $g$ & method & $\varepsilon$ Lap. & $\varepsilon$ Gaus. & $\varepsilon$ Lap. & $\varepsilon$ Gaus. \\
\hline \multirow{2}{*}{ (a) or \#2 } & DG, lower median & 0.015 & 0.018 & - & - \\
$\chi^{2}(3)$ & DG, higher median & 0.018 & 0.022 & - & - \\
\cline { 2 - 6 }$(s 2 n=4)$ & Proj.: median & 0.014 & 0.016 & - & - \\
& Proj.: mean & 0.015 & 0.018 & - & - \\
\hline \multirow{2}{*}{ (c) or \#6 } & DG, lower median & - & - & 0.0021 & 0.0023 \\
Mix.Gamma & DG, higher median & - & - & 0.0024 & 0.0026 \\
\cline { 2 - 6 }$(s 2 n=10)$ & Proj.: median & - & - & 0.0017 & 0.0020 \\
& Proj., mean & - & - & 0.0019 & 0.0021 \\
\hline \multirow{2}{*}{ (e) or \#1 } & DG, lower median & 0.0071 & 0.0080 & 0.0041 & 0.0051 \\
Gauss & DG, higher median & 0.011 & 0.012 & 0.0059 & 0.0072 \\
\cline { 2 - 6 }$(s 2 n=4)$ & Proj.: median & 0.012 & 0.017 & 0.0049 & 0.0066 \\
& Proj.: mean & 0.016 & 0.022 & 0.0059 & 0.0088 \\
\hline \multirow{2}{*}{ (f) or \#3 } & DG, lower median & 0.018 & 0.027 & 0.011 & 0.020 \\
Mix.Gauss & DG, higher median & 0.031 & 0.034 & 0.023 & 0.028 \\
\cline { 2 - 6 }$(s 2 n=4)$ & Proj.: median & 0.016 & 0.022 & 0.0063 & 0.0088 \\
& Proj.: mean & 0.017 & 0.024 & 0.0071 & 0.010 \\
\hline
\end{tabular}

Table 4: Ratio between MISE with misspecified error density (Laplace errors, $g$ estimated as if errors were Gaussian and reciprocally) and MISE with correctly specified error density.

\begin{tabular}{cccccccccc}
\hline \multicolumn{1}{c}{$\times 10^{-2}$} & \multicolumn{2}{c}{$n=1000$} & \multicolumn{2}{c}{$n=5000$} & \multicolumn{2}{c}{$n=10000$} & \multicolumn{2}{c}{$n=25000$} \\
\hline$g$ & $s 2 n$ & $\varepsilon$ Lap. & $\varepsilon$ Gaus. & $\varepsilon$ Lap. & $\varepsilon$ Gaus. & $\varepsilon$ Lap. & $\varepsilon$ Gaus. & $\varepsilon$ Lap. & $\varepsilon$ Gaus. \\
\hline Lapl. & 2 & 1.6 & 1.4 & 2.2 & 1.8 & 2.3 & 2.9 & 2.4 & 4.5 \\
& 4 & 1 & 1.3 & 1 & 1.9 & 1 & 2.2 & 1 & 2.3 \\
\hline Mix.Gam. & 2 & 1 & 1.1 & 1.3 & 1.6 & 1.6 & 2.1 & 2.2 & 3 \\
& 4 & 1 & 1 & 1.1 & 1.2 & 1 & 1.3 & 1.1 & 1.5 \\
\hline Cauchy & 2 & 1.3 & 1.3 & 1.7 & 1.6 & 2.5 & 1.2 & 3.7 & 1.5 \\
& 4 & 1.1 & 1 & 1.2 & 1.1 & 1.3 & 1.1 & 1.4 & 1.2 \\
\hline Gauss & 2 & 1.1 & 1.4 & 1.4 & 1.1 & 2 & 1 & 3.1 & 1.2 \\
& 4 & 1 & 0.81 & 1.2 & 1 & 1.2 & 1 & 1.8 & 1.3 \\
\hline
\end{tabular}

Concluding remarks : Our estimation procedure provides an adaptive estimator which achieves the minimax rate of convergence (up to a possible logarithmic factor) in all the cases where lower bounds are available, without any prior smoothness knowledge on the unknown density $g$. In 
particular it solves almost in the best way the bias-variance problem when the best compromise would not be easily computable. Furthermore, this estimation procedure induces a fast practical algorithm with pretty good practical results.

\section{PROOFS}

\subsection{Proof of Proposition 1.}

According to (1), for any given $m$ belonging to $\mathcal{M}_{n}, \hat{g}_{m}^{(n)}$ satisfies, $\gamma_{n}\left(\hat{g}_{m}^{(n)}\right)-\gamma_{n}\left(g_{m}^{(n)}\right) \leq 0$. Denoting by $\nu_{n}(t)$ the centered empirical process

$$
\nu_{n}(t)=\frac{1}{n} \sum_{i=1}^{n}\left[u_{t}^{*}\left(Z_{i}\right)-\langle t, g\rangle\right]
$$

we have that

$$
\gamma_{n}(t)-\gamma_{n}(s)=\|t-g\|^{2}-\|s-g\|^{2}-2 \nu_{n}(t-s),
$$

and therefore, $\left\|g-\hat{g}_{m}^{(n)}\right\|^{2} \leq\left\|g-g_{m}^{(n)}\right\|^{2}+2 \nu_{n}\left(\hat{g}_{m}^{(n)}-g_{m}^{(n)}\right)$. Since $\hat{a}_{m, j}-a_{m, j}=\nu_{n}\left(\varphi_{m, j}\right)$, we get that

$$
\nu_{n}\left(\hat{g}_{m}^{(n)}-g_{m}^{(n)}\right)=\sum_{|j| \leq K_{n}}\left(\hat{a}_{m, j}-a_{m, j}\right) \nu_{n}\left(\varphi_{m, j}\right)=\sum_{|j| \leq K_{n}}\left[\nu_{n}\left(\varphi_{m, j}\right)\right]^{2},
$$

and consequently $\mathbb{E}\left\|g-\hat{g}_{m}^{(n)}\right\|^{2} \leq\left\|g-g_{m}^{(n)}\right\|^{2}+2 \sum_{j \in \mathbb{Z}} \operatorname{Var}\left[\nu_{n}\left(\varphi_{m, j}\right)\right]$. Now, since the $X_{i}$ 's and the $\varepsilon_{i}$ 's are independent and identically distributed random variables, we get that $\operatorname{Var}\left[\nu_{n}\left(\varphi_{m, j}\right)\right]=$ $n^{-2} \sum_{i=1}^{n} \operatorname{Var}\left[u_{\varphi_{m, j}}^{*}\left(Z_{i}\right)\right]=n^{-1} \operatorname{Var}\left[u_{\varphi_{m, j}}^{*}\left(Z_{1}\right)\right]$.

Apply Lemma 2 to get that $\sum_{j \in \mathbb{Z}} \operatorname{Var}\left[\nu_{n}\left(\varphi_{m, j}\right)\right] \leq \Delta_{1}(m) / n$, where $\Delta_{1}(m)$ is defined in Proposition 1. It remains to study $\left\|g-g_{m}^{(n)}\right\|^{2}$. By applying Pythagoras Theorem, we have $\left\|g-g_{m}^{(n)}\right\|^{2}=\|$ $g-g_{m}\left\|^{2}+\right\| g_{m}-g_{m}^{(n)} \|^{2}$, where $\left\|g_{m}-g_{m}^{(n)}\right\|^{2}=\sum_{|j|>K_{n}} a_{m, j}^{2} \leq\left(\sup _{j} j a_{m, j}\right)^{2} \sum_{|j|>K_{n}} j^{-2}$. Now we write that

$$
\begin{aligned}
j a_{m, j} & =j \sqrt{L_{m}} \int \varphi\left(L_{m} x-j\right) g(x) d x \\
& \leq L_{m}^{3 / 2} \int|x|\left|\varphi\left(L_{m} x-j\right)\right| g(x) d x+\sqrt{L_{m}} \int\left|L_{m} x-j \| \varphi\left(L_{m} x-j\right)\right| g(x) d x \\
& \leq L_{m}^{3 / 2}\left(\int\left|\varphi\left(L_{m} x-j\right)\right|^{2} d x\right)^{1 / 2}\left(\int x^{2} g^{2}(x) d x\right)^{1 / 2}+\sqrt{L_{m}} \sup _{x}|x \varphi(x)| .
\end{aligned}
$$

This implies finally that $j a_{m, j} \leq L_{m}\left(M_{2}\right)^{1 / 2}+\sqrt{L_{m}}$, and Proposition 1 follows.

\subsection{Proof of Theorem 1}

By definition, $\tilde{g}$ satisfies that for all $m \in \mathcal{M}_{n}, \gamma_{n}(\tilde{g})+\operatorname{pen}(\hat{m}) \leq \gamma_{n}\left(g_{m}^{(n)}\right)+\operatorname{pen}(m)$. Therefore, by applying (14) we get that

$$
\|\tilde{g}-g\|^{2} \leq\left\|g_{m}^{(n)}-g\right\|^{2}+2 \nu_{n}\left(\tilde{g}-g_{m}^{(n)}\right)+\operatorname{pen}(m)-\operatorname{pen}(\hat{m}) .
$$

Next, we use that if $t=t_{1}+t_{2}$ with $t_{1}$ in $S_{m}^{(n)}$ and $t_{2}$ in $S_{m^{\prime}}^{(n)}$, then $t$ is such that $t^{*}$ has its support in $\left[-\pi L_{\max \left(m, m^{\prime}\right)}, \pi L_{\max \left(m, m^{\prime}\right)}\right]$ and therefore $t$ belongs to $S_{\max \left(m, m^{\prime}\right)}^{(n)}$. If we denote by $B_{m, m^{\prime}}(0,1)$ the 
set $B_{m, m^{\prime}}(0,1)=\left\{t \in S_{\max \left(m, m^{\prime}\right)}^{(n)} /\|t\|=1\right\}$, then $\left|\nu_{n}\left(\tilde{g}-g_{m}^{(n)}\right)\right| \leq\left\|\tilde{g}-g_{m}^{(n)}\right\| \sup _{t \in B_{m, m}(0,1)}\left|\nu_{n}(t)\right|$. Consequently, by using that $2 u v \leq a^{-1} u^{2}+a v^{2}$, for $a>1$, we get

$$
\|\tilde{g}-g\|^{2} \leq\left\|g_{m}^{(n)}-g\right\|^{2}+a^{-1}\left\|\tilde{g}-g_{m}^{(n)}\right\|^{2}+a \sup _{t \in B_{m, \hat{m}}(0,1)} \nu_{n}^{2}(t)+\operatorname{pen}(m)-\operatorname{pen}(\hat{m})
$$

and therefore, by writing that $\left\|\tilde{g}-g_{m}^{(n)}\right\|^{2} \leq\left(1+y^{-1}\right)\|\tilde{g}-g\|^{2}+(1+y)\left\|g-g_{m}^{(n)}\right\|^{2}$, with $y=$ $(a+1) /(a-1)$ for $a>1$, we infer that

$$
\|\tilde{g}-g\|^{2} \leq\left(\frac{a+1}{a-1}\right)^{2}\left\|g-g_{m}^{(n)}\right\|^{2}+\frac{a(a+1)}{a-1} \sup _{t \in B_{m, \hat{m}}(0,1)} \nu_{n}^{2}(t)+\frac{a+1}{a-1}(\operatorname{pen}(m)-\operatorname{pen}(\hat{m})) .
$$

Choose some positive function $p\left(m, m^{\prime}\right)$ such that $a p\left(m, m^{\prime}\right) \leq \operatorname{pen}(m)+\operatorname{pen}\left(m^{\prime}\right)$. Consequently, for $\kappa_{a}=(a+1) /(a-1)$ we have

$$
\begin{aligned}
& \|\tilde{g}-g\|^{2} \leq \kappa_{a}^{2}\left[\left\|g-g_{m}\right\|^{2}+\left\|g_{m}-g_{m}^{(n)}\right\|^{2}+\operatorname{pen}(m)\right]+a \kappa_{a} W_{n}(\hat{m}) \\
& \text { with } \\
& \qquad W_{n}\left(m^{\prime}\right):=\left[\sup _{t \in B_{m, m^{\prime}}(0,1)}\left|\nu_{n}(t)\right|^{2}-p\left(m, m^{\prime}\right)\right]_{+},
\end{aligned}
$$

that is, according to the proof of Proposition 1,

$$
\|\tilde{g}-g\|^{2} \leq \kappa_{a}^{2}\left\|g-g_{m}\right\|^{2}+\kappa_{a}^{2}\left(M_{2}+1\right)\left(\pi L_{m}\right)^{2} / K_{n}+2 \kappa_{a} \operatorname{pen}(m)+a \kappa_{a} \sum_{m^{\prime} \in \mathcal{M}_{n}} W_{n}\left(m^{\prime}\right) .
$$

The main point of the proof lies in studying $W_{n}\left(m^{\prime}\right)$, and more precisely in finding $p\left(m, m^{\prime}\right)$ such that for a constant $K$,

$$
\sum_{m^{\prime} \in \mathcal{M}_{n}} \mathbb{E}\left(W_{n}\left(m^{\prime}\right)\right) \leq K / n .
$$

In this case, combining (16) and (17) we infer that, for all $m$ in $\mathcal{M}_{n}$,

$$
\mathbb{E}\|g-\tilde{g}\|^{2} \leq \kappa_{a}^{2}\left\|g-g_{m}\right\|^{2}+\kappa_{a}^{2}\left(M_{2}+1\right)\left(\pi L_{m}\right)^{2} / K_{n}+2 \kappa_{a} \operatorname{pen}(m)+a \kappa_{a} K / n,
$$

which can also be written

$$
\mathbb{E}\|g-\tilde{g}\|^{2} \leq C_{a} \inf _{m \in \mathcal{M}_{n}}\left[\left\|g-g_{m}\right\|^{2}+\operatorname{pen}(m)+\left(M_{2}+1\right)\left(\pi L_{m}\right)^{2} / K_{n}\right]+a \kappa_{a} K / n,
$$

where $C_{a}=\max \left(\kappa_{a}^{2}, 2 \kappa_{a}\right)$ suits. It remains thus to find $p\left(m, m^{\prime}\right)$ such that (17) holds. This will be done by applying the following immediate integration of Talagrand's Inequality (see Talagrand (1996)):

Lemma 1. Let $Y_{1}, \ldots, Y_{n}$ be i.i.d. random variables and $r_{n}(f)=(1 / n) \sum_{i=1}^{n}\left[f\left(Y_{i}\right)-\mathbb{E}\left(f\left(Y_{i}\right)\right)\right]$ for $f$ belonging to a countable class $\mathcal{F}$ of uniformly bounded measurable functions. Then for $\xi^{2}>0$

$$
\mathbb{E}\left[\sup _{f \in \mathcal{F}}\left|r_{n}(f)\right|^{2}-2\left(1+2 \xi^{2}\right) H^{2}\right]_{+} \leq \frac{6}{K_{1}}\left(\frac{v}{n} e^{-K_{1} \xi^{2} \frac{n H^{2}}{v}}+\frac{8 M_{1}^{2}}{K_{1} n^{2} C^{2}\left(\xi^{2}\right)} e^{-\frac{K_{1} C(\xi) \xi}{\sqrt{2}} \frac{n H}{M_{1}}}\right),
$$

with $C(\xi)=\sqrt{1+\xi^{2}}-1, K_{1}$ is a universal constant, and where

$$
\sup _{f \in \mathcal{F}}\|f\|_{\infty} \leq M_{1}, \quad \mathbb{E}\left[\sup _{f \in \mathcal{F}}\left|r_{n}(f)\right|\right] \leq H, \quad \sup _{f \in \mathcal{F}} \operatorname{Var}\left(f\left(Y_{1}\right)\right) \leq v .
$$


Usual density arguments show that this result can be applied to the class of functions $\mathcal{F}=$ $B_{m, m^{\prime}}(0,1)$. Let us denote by $m^{*}=\max \left(m, m^{\prime}\right)$. Combining Lemma 3 and Lemma 4 , we propose to take

$$
H^{2}=H^{2}\left(m^{*}\right)=\lambda_{1} L_{m^{*}}^{2 \gamma+1-\delta} \exp \left\{2 \mu \sigma^{\delta}\left(\pi L_{m^{*}}\right)^{\delta}\right\} / n \text { and } \quad M_{1}=\sqrt{n H^{2}},
$$

where $\lambda_{1}=\lambda_{1}\left(\gamma, \kappa_{0}, \mu, \sigma, \delta\right)$ is defined by (4). Again, by applying Lemma 4 , we take $v \geq \Delta_{2}\left(m^{*}, h\right)$ with

$$
\Delta_{2}(m, h)=L_{m}^{2} \iint\left|\frac{\varphi^{*}(x) \varphi^{*}(y)}{f_{\varepsilon}^{*}\left(\sigma L_{m} x\right) f_{\varepsilon}^{*}\left(\sigma L_{m} y\right)} h^{*}\left(L_{m}(x-y)\right)\right|^{2} d x d y .
$$

For $\delta>1$ we use a rough bound for $\Delta_{2}(m, h)$ given by $\sqrt{\Delta_{2}\left(m^{*}, h\right)} \leq 2 \pi n H^{2}$. When $\delta \leq 1$, write that

$$
\begin{aligned}
\Delta_{2}(m, h) & \leq \kappa_{0}^{-2} L_{m}^{2}\left(1+\left(\sigma \pi L_{m}\right)^{2}\right)^{\gamma} \exp \left\{2 \mu \sigma^{\delta}\left(\pi L_{m}\right)^{\delta}\right\} \int_{-\pi}^{\pi} \frac{d x}{\left|f_{\varepsilon}^{*}\left(\sigma L_{m} x\right)\right|^{2}} \int\left|h^{*}\left(L_{m} u\right)\right|^{2} d u \\
& \leq 2 \kappa_{0}^{-2} \pi \lambda_{1}\left(1+\sigma^{2} \pi^{2}\right)^{\gamma}\left\|h^{*}\right\|^{2} L_{m}^{4 \gamma+1-\delta} \exp \left\{4 \mu \sigma^{\delta}\left(\pi L_{m}\right)^{\delta}\right\}
\end{aligned}
$$

Using that $\left\|h^{*}\right\|^{2} \leq\left\|f_{\varepsilon}^{*}\right\|^{2}<\infty$, we take $v=\lambda_{2} L_{m^{*}}^{2 \gamma+\min (1 / 2-\delta / 2,1-\delta)} \exp \left\{2 \mu \sigma^{\delta}\left(\pi L_{m^{*}}\right)^{\delta}\right\}$, where $\lambda_{2}=\lambda_{2}\left(\gamma, \kappa_{0}, \mu, \sigma, \delta\right)$ is defined in Theorem 1. From the definition (15) of $W_{n}\left(m^{\prime}\right)$, by taking $p\left(m, m^{\prime}\right)=2\left(1+2 \xi^{2}\right) H^{2}$, we get that

$$
\mathbb{E}\left(W_{n}\left(m^{\prime}\right)\right) \leq \mathbb{E}\left[\sup _{t \in B_{m, m^{\prime}}(0,1)}\left|\nu_{n}(t)\right|^{2}-2\left(1+2 \xi^{2}\right) H^{2}\right]_{+} \cdot
$$

By applying (18), we get the global bound $\mathbb{E}\left(W_{n}\left(L_{m^{\prime}}\right)\right) \leq K\left[I\left(L_{m^{*}}\right)+I I\left(m^{*}\right)\right]$, where $I\left(m^{*}\right)$ and $I I\left(m^{*}\right)$ are defined by

$$
\begin{aligned}
I\left(m^{*}\right) & =\frac{\lambda_{2} L_{m^{*}}^{2 \gamma+\min (1 / 2-\delta / 2,1-\delta)} \exp \left\{2 \mu \sigma^{\delta}\left(\pi L_{m^{*}}\right)^{\delta}\right\}}{n} \exp \left\{-K_{1} \xi^{2}\left(\lambda_{1} / \lambda_{2}\right) L_{m^{*}}^{(1 / 2-\delta / 2)_{+}}\right\} \\
\text {and } \quad I I\left(m^{*}\right) & =\frac{\lambda_{1} L_{m^{*}}^{2 \gamma+1-\delta} e^{2 \mu \sigma^{\delta}\left(\pi L_{m^{*}}\right)^{\delta}}}{n^{2}} \exp \left\{-K_{1} \xi C(\xi) \sqrt{n} / \sqrt{2}\right\},
\end{aligned}
$$

with $\lambda_{2}=\lambda_{2}\left(\gamma, \kappa_{0}, \mu, \sigma, \delta\right)$ defined in Theorem 1 .

- Study of $\sum_{m^{\prime} \in \mathcal{M}_{n}} I I\left(m^{*}\right)$. We have $\sum_{m^{\prime} \in \mathcal{M}_{n}} I I\left(m^{*}\right) \leq\left|\mathcal{M}_{n}\right| \exp \left\{-K_{1} \xi C(\xi) \sqrt{n} / \sqrt{2}\right\} 2 \lambda_{1} \Gamma\left(m_{n}\right) / n^{2}$, according to the choices for $v, H^{2}$ and $M_{1}$. Consequently, since under (6), $\Gamma\left(m_{n}\right) / n$ is bounded, $\sum_{m^{\prime} \in \mathcal{M}_{n}} I I\left(m^{*}\right) \leq C / n$.

- Study of $\sum_{m^{\prime} \in \mathcal{M}_{n}} I\left(m^{*}\right)$. Denote by $\psi=2 \gamma+\min (1 / 2-\delta / 2,1-\delta), \omega=(1 / 2-\delta / 2)_{+}$, $K^{\prime}=K_{1} \lambda_{1} / \lambda_{2}$, then for $a, b \geq 1$, we infer that

$$
\begin{aligned}
& \max (a, b)^{\psi} e^{2 \mu \sigma^{\delta} \pi^{\delta} \max (a, b)^{\delta}} e^{-K^{\prime} \xi^{2} \max (a, b)^{\omega}} \leq\left(a^{\psi} e^{2 \mu \sigma^{\delta} \pi^{\delta} a^{\delta}}+b^{\psi} e^{2 \mu \sigma^{\delta} \pi^{\delta} b^{\delta}}\right) e^{-\left(K^{\prime} \xi^{2} / 2\right)\left(a^{\omega}+b^{\omega}\right)} \\
& \leq a^{\psi} e^{2 \mu \sigma^{\delta} \pi^{\delta} a^{\delta}} e^{-\left(K^{\prime} \xi^{2} / 2\right) a^{\omega}} e^{-\left(K^{\prime} \xi^{2} / 2\right) b^{\omega}}+b^{\psi} e^{2 \mu \sigma^{\delta} \pi^{\delta} b^{\delta}} e^{-\left(K^{\prime} \xi^{2} / 2\right) b^{\omega}} .
\end{aligned}
$$

Consequently, if we denote by $\tilde{\Gamma}$ the quantity $\tilde{\Gamma}(m)=L_{m}^{2 \gamma+\min (1 / 2-\delta / 2,1-\delta)} \exp \left\{2 \mu \sigma^{\delta}\left(\pi L_{m}\right)^{\delta}\right\}$ then

$$
\begin{aligned}
\sum_{m^{\prime} \in \mathcal{M}_{n}} I\left(m^{*}\right) \leq & \frac{2 \lambda_{2} \tilde{\Gamma}(m)}{n} \exp \left\{-\left(K^{\prime} \xi^{2} / 2\right)\left(L_{m}\right)^{(1 / 2-\delta / 2)_{+}}\right\} \sum_{m^{\prime} \in \mathcal{M}_{n}} \exp \left\{-\left(K^{\prime} \xi^{2} / 2\right)\left(L_{m^{\prime}}\right)^{(1 / 2-\delta / 2)_{+}}\right\} \\
& +\sum_{m^{\prime} \in \mathcal{M}_{n}} \frac{2 \lambda_{2} \tilde{\Gamma}\left(m^{\prime}\right)}{n} \exp \left\{-\left(K^{\prime} \xi^{2} / 2\right)\left(L_{m^{\prime}}\right)^{(1 / 2-\delta / 2)_{+}}\right\} .
\end{aligned}
$$


1) Case $0 \leq \delta<1 / 3$ In that case, since $\delta<(1 / 2-\delta / 2)_{+}$, the choice $\xi^{2}=1$ ensures that $\tilde{\Gamma}(m) \exp \left\{-\left(K^{\prime} \xi^{2} / 2\right)\left(L_{m}\right)^{(1 / 2-\delta / 2)}\right\}$ is bounded and thus the first term in (21) is bounded by $C / n$. Since $1 \leq m \leq m_{n}$ with $m_{n}$ satisfying (6), $\sum_{m^{\prime} \in \mathcal{M}_{n}}\left(\tilde{\Gamma}\left(m^{\prime}\right) / n\right) \exp \left\{-\left(K^{\prime} / 2\right)\left(L_{m^{\prime}}\right)^{(1 / 2-\delta / 2)}\right\}$ is bounded by $\tilde{\tilde{C}} / n$, and hence $\sum_{m^{\prime} \in \mathcal{M}_{n}} I\left(m^{*}\right) \leq C / n$. Consequently, (17) hold if we choose $\operatorname{pen}(m)=2 a\left(1+2 \xi^{2}\right) \lambda_{1}\left(L_{m}\right)^{2 \gamma+1-\delta} \exp \left\{2 \mu \sigma^{\delta}\left(\pi L_{m}\right)^{\delta}\right\} / n$.

2) Case $\delta=1 / 3 \quad$ According to the inequality (20), $\xi^{2}$ is such that $2 \mu \sigma^{\delta} \pi^{\delta}\left(L_{m^{*}}\right)^{\delta}-\left(K^{\prime} \xi^{2} / 2\right) L_{m^{*}}^{\delta}=$ $-2 \mu \sigma^{\delta}\left(\pi L_{m^{*}}\right)^{\delta}$ that is $\xi^{2}=\left(4 \mu \sigma^{\delta} \pi^{\delta} \lambda_{2}\right) /\left(K_{1} \lambda_{1}\right)$. Arguing as for the case $0 \leq \delta<1 / 3$, this choice ensures that $\sum_{m^{\prime} \in \mathcal{M}_{n}} I\left(m^{*}\right) \leq C / n$, and consequently (17) holds. The result follows for $p\left(m, m^{\prime}\right)=$ $2\left(1+2 \xi^{2}\right) \lambda_{1} L_{m^{*}}^{2 \gamma+1-\delta} \exp \left(2 \mu \sigma^{\delta}\left(\pi L_{m^{*}}\right)^{\delta}\right) / n$, and $\operatorname{pen}(m)=2 a\left(1+2 \xi^{2}\right) \lambda_{1} L_{m}{ }^{2 \gamma+1-\delta} \exp \left(2 \mu \sigma^{\delta}\left(\pi L_{m}\right)^{\delta}\right) / n$.

3) Case $\delta>1 / 3$ If $\delta>(1 / 2-\delta / 2)_{+}$, according to (20) we choose $\xi^{2}=\xi^{2}\left(L_{m}, L_{m^{\prime}}\right)$ such that $2 \mu \sigma^{\delta} \pi^{\delta}\left(L_{m^{*}}\right)^{\delta}-\left(K^{\prime} \xi^{2} / 2\right) L_{m^{*}}^{\omega}=-2 \mu \sigma^{\delta} \pi^{\delta}\left(L_{m^{*}}\right)^{\delta}$ that is $\xi^{2}=\xi^{2}\left(m, m^{\prime}\right)=\left(4 \mu \sigma^{\delta} \pi^{\delta} \lambda_{2}\right) /\left(K_{1} \lambda_{1}\right) L_{m^{*}}^{\delta-\omega}$. This choice ensures that $\sum_{m^{\prime} \in \mathcal{M}_{n}} I\left(m^{*}\right) \leq C / n$, and consequently (17) holds if $p\left(m, m^{\prime}\right)=$ $2\left(1+2 \xi^{2}\left(m, m^{\prime}\right)\right) \lambda_{1} L_{m^{*}}^{2 \gamma+1-\delta} \exp \left(2 \mu \sigma^{\delta}\left(\pi L_{m^{*}}\right)^{\delta}\right) / n$, associated to the penalty $\operatorname{pen}(m)=2 a(1+$ $\left.2 \xi^{2}\left(L_{m}, m\right)\right) \lambda_{1}\left(L_{m}\right)^{2 \gamma+1-\delta} \exp \left(2 \mu \sigma^{\delta}\left(\pi L_{m}\right)^{\delta}\right) / n$.

\subsection{Technical Lemmas}

Lemma 2. Let $\nu_{n}(t)$ be defined by (10), $\Delta_{1}(m)$ be defined in Proposition 1. Under Assumptions $\left(\left(\mathrm{A}_{1}^{X, \varepsilon}\right)\right.$

$$
\left\|\sum_{j \in \mathbb{Z}}\left|u_{\varphi_{m, j}}^{*}\right|^{2}\right\|_{\infty} \leq \Delta_{1}(m), \text { and } \sup _{g \in \mathcal{S}_{s, r, b}\left(C_{1}\right)} \sum_{j \in \mathbb{Z}} \operatorname{Var}\left[\nu_{n}\left(\varphi_{m, j}\right)\right] \leq \Delta_{1}(m) / n .
$$

Proof of Lemma 2 Use the definition of $u_{\varphi_{m, j}}^{*}(z)$ to get that

$\sum_{j \in \mathbb{Z}}\left|u_{\varphi_{m, j}}^{*}(z)\right|^{2}=\sum_{j \in \mathbb{Z}}\left|\int \exp \{i x z\} u_{\varphi_{m, j}}(x) d x\right|^{2}=\frac{L_{m}}{(2 \pi)^{2}} \sum_{j \in \mathbb{Z}}\left|\int \exp \left\{-i x z L_{m}\right\} \exp \{i j x\} \frac{\varphi^{*}(x)}{f_{\varepsilon}^{*}\left(x L_{m} \sigma\right)} d x\right|^{2}$.

By Parseval's Formula,

$$
\sum_{j \in \mathbb{Z}}\left|u_{\varphi_{m, j}}^{*}(z)\right|^{2}=(2 \pi)^{-1} L_{m} \int\left|\frac{\varphi^{*}(x)}{f_{\varepsilon}^{*}\left(x L_{m} \sigma\right)}\right|^{2} d x=\Delta_{1}(m),
$$

which entails that the first part of the bound (22) is proved. The second part follows since $\sum_{j \in \mathbb{Z}} \operatorname{Var}\left[\nu_{n}\left(\varphi_{m, j}\right)\right] \leq n^{-1} \int \sum_{j \in \mathbb{Z}}\left|u_{\varphi_{m, j}}^{*}(z)\right|^{2} h(z) d z$.

Lemma 3. Let $\Delta_{1}(m)$ and $R(\mu, \delta, \sigma)$ be defined in Proposition 1 and in (4). Then under the assumption $\left(\mathrm{A}_{2}^{\varepsilon}\right), \Delta_{1}(m) \leq \frac{1}{\pi \kappa_{0}^{2} R(\mu, \delta, \sigma)}\left(\pi L_{m}\right)^{1-\delta}\left(\sigma^{2} L_{m}^{2} \pi^{2}+1\right)^{\gamma} \exp \left\{2 \mu \sigma^{\delta} \pi^{\delta} L_{m}^{\delta}\right\}$.

Proof of Lemma 3. Under the assumption $\left(\mathrm{A}_{2}^{\varepsilon}\right), \Delta_{1}(m) \leq\left(\pi \kappa_{0}^{2}\right)^{-1}\left(\sigma^{2} L_{m}^{2} \pi^{2}+1\right)^{\gamma} \int_{0}^{\pi L_{m}} \exp \left\{2 \mu \sigma^{\delta} u^{\delta}\right\} d u$. If $\delta=0$, by convention $\mu=0$, and hence the integral in the previous bound is less than $\pi L_{m}$.

Consider now the case $0<\delta \leq 1$. Easy calculations provide that

$$
\int_{0}^{\pi L_{m}} e^{2 \mu \sigma^{\delta} u^{\delta}} d u=\int_{0}^{\pi L_{m}}\left(2 \mu \sigma^{\delta} \delta u^{\delta-1} e^{2 \mu \sigma^{\delta} u^{\delta}}\right) \frac{d u}{2 \mu \sigma^{\delta} \delta u^{\delta-1}} \leq \frac{\left(\pi L_{m}\right)^{1-\delta}}{2 \mu \sigma^{\delta} \delta}\left[e^{2 \mu \sigma^{\delta} u^{\delta}}\right]_{0}^{\pi L_{m}}
$$


and therefore $\int_{0}^{\pi L_{m}} \exp \left\{2 \mu \sigma^{\delta} u^{\delta}\right\} d u \leq\left[\left(\pi L_{m}\right)^{1-\delta} /\left(2 \mu \sigma^{\delta} \delta\right)\right] \exp \left(2 \mu \sigma^{\delta}\left(\pi L_{m} \sigma\right)^{\delta}\right)$.

Now, if $\delta>1$, then by using that $u^{\delta}=u^{\delta-1} u$, and consequently Lemma 3 follows from

$$
\int_{0}^{\pi L_{m}} \exp \left\{2 \mu \sigma^{\delta} u^{\delta}\right\} d u \leq \int_{0}^{\pi L_{m}} \exp \left\{2 \mu \sigma^{\delta}\left(\pi L_{m}\right)^{\delta-1} u\right\} d u \leq \frac{\left(\pi L_{m}\right)^{1-\delta}}{2 \mu \sigma^{\delta}} \exp \left(2 \mu \sigma^{\delta}\left(\pi L_{m}\right)^{\delta}\right) . \square
$$

Lemma 4. Let $\nu_{n}(t), \Delta_{1}(m)$ and $\Delta_{2}(m, h)$ be defined in (13), Proposition 1 and in (19). Then under $\left(\mathrm{A}_{1}^{X, \varepsilon}\right)$

$$
\begin{gathered}
\sup _{t \in B_{m, m^{\prime}}(0,1)}\left\|u_{t}^{*}\right\|_{\infty} \leq \sqrt{\Delta_{1}\left(m^{*}\right)} \quad \mathbb{E}\left[\sup _{t \in B_{m, m^{\prime}}(0,1)}\left|\nu_{n}(t)\right|\right] \leq \sqrt{\Delta_{1}\left(m^{*}\right) / n}, \\
\quad \text { and } \sup _{t \in B_{m, m^{\prime}}(0,1)} \operatorname{Var}\left(u_{t}^{*}\left(Z_{1}\right)\right) \leq \sqrt{\Delta_{2}\left(m^{*}, h\right)} /(2 \pi) .
\end{gathered}
$$

Proof of Lemma 4 By combining Cauchy-Schwarz Inequality and (23), the square of the first term $\sup _{t \in B_{m, m^{\prime}}(0,1)}\left\|u_{t}^{*}\right\|_{\infty}^{2}$ is bounded by $\sum_{j \in \mathbb{Z}} \int \mid \varphi_{m^{*}, j}^{*}(u) / f_{\varepsilon}^{*}\left(\left.\sigma u\right|^{2} d u=\Delta_{1}\left(m^{*}\right)\right.$. Now, we have $\mathbb{E}\left[\sup _{t \in B_{m, m^{\prime}}(0,1)}\left|\nu_{n}(t)\right|\right] \leq \mathbb{E}\left[\left(\sum_{j \in \mathbb{Z}}\left(\nu_{n}\left(\varphi_{m^{*}, j}\right)\right)^{2}\right)^{1 / 2}\right] \leq\left[\sum_{j \in \mathbb{Z}} \operatorname{Var}\left(\nu_{n}\left(\varphi_{m^{*}, j}\right)\right)\right]^{1 / 2}$, which is bounded, by applying the second part of $(22)$ in Lemma 2 , by $\sqrt{\Delta_{1}\left(m^{*}\right) / n}$. Now write that $\sup _{t \in B_{m, m^{\prime}}(0,1)} \operatorname{Var}\left(u_{t}^{*}\left(Z_{1}\right)\right) \leq \sup _{t \in B_{m, m^{\prime}}(0,1)} \mathbb{E}\left[\left|u_{t}^{*}\left(Z_{1}\right)\right|^{2}\right] \leq\left[\sum_{j, k \in \mathbb{Z}}\left|Q_{j, k}\left(m^{*}\right)\right|^{2}\right]^{1 / 2}$, with $Q_{j, k}(m)=$ $\mathbb{E}\left[u_{\varphi_{m, j}}^{*}\left(Z_{1}\right) u_{\varphi_{m, k}}^{*}\left(-Z_{1}\right)\right]$ also given by

$$
Q_{j, k}(m)=\frac{L_{m}}{(2 \pi)^{2}} \iint \exp \{i j x-i k y\} \frac{\varphi^{*}(x) \varphi^{*}(y)}{f_{\varepsilon}^{*}\left(\sigma L_{m} x\right) f_{\varepsilon}^{*}\left(\sigma L_{m} y\right)} h^{*}\left(L_{m}(x-y)\right) d x d y .
$$

Apply Parseval's Formula to get the result since

$$
\sum_{j, k \in \mathbb{Z}}\left|Q_{j, k}(m)\right|^{2}=\frac{L_{m}^{2}}{(2 \pi)^{2}} \iint\left|\frac{\varphi^{*}(x) \varphi^{*}(y)}{f_{\varepsilon}^{*}\left(\sigma L_{m} x\right) f_{\varepsilon}^{*}\left(\sigma L_{m} y\right)} h^{*}\left(L_{m}(x-y)\right)\right|^{2} d x d y . \square
$$

ACKNOWLEDGEMENTS We thank Cristina Butucea and Alexander Tsybakov for helpful discussions on this subject.

\section{REFERENCES}

A.R. Barron, L. Birgé \& P. Massart (1999). Risk bounds for model selection via penalization. Probability Theory and Related Fields, 113, 301-413.

L. Birgé \& Y. Rozenholc (2005). How many bins must be put in a regular histogram. To appear in ESAIM, Probability and Statistics.

C. Butucea (2004). Deconvolution of supersmooth densities with smooth noise. The Canadian Journal of Statistics, 32, 181-192.

C. Butucea \& A.B. Tsybakov (2004). Sharp optimality and some effects of dominating bias in density deconvolution, Preprint LPMA-898, http://www.proba.jussieu.fr/mathdoc/preprints/index.html\#2004.

C. Butucea \& C. Matias (2005). Minimax estimation of the noise level and of the deconvolution density in a semiparametric convolution model. Bernoulli, 11, 309-340. 
R.J. Carroll, \& P. Hall (1988). Optimal rates of convergence for deconvolving a density. Journal of the American Statistical Association, 83, 1184-1186.

E.A. Cator (2001). Deconvolution with arbitrarily smooth kernels. Statistics and Probability Letters, $54,205-214$.

L. Cavalier, Y. Golubev, O. Lepski \& A.B. Tsybakov (2003). Block thresholding and sharp adaptive estimation in severely ill-posed inverse problems. Theory Prob. Appl., 48.

F. Comte and V. Genon-Catalot (2005) Penalized projection estimator for volatility density. Preprint MAP5 2005-9, http://www.math-info.univ-paris5.fr/map5/publis/titres05.html.

A. Delaigle \& I. Gijbels (2004a). Practical bandwidth selection in deconvolution kernel density estimation. Computational Statistics and Data Analysis, 45, 249-267.

A. Delaigle \& I. Gijbels (2004b). Bootstrap bandwidth selection in kernel density estimation from a contaminated sample. Annals of the Institute of Statistical Mathematics, 56, 19-47.

L. Devroye (1986). Non-Uniform Random Variable Generation. Springer-Verlag, New-York.

L. Devroye (1989). Consistent deconvolution in density estimation. The Canadian Journal of Statistics, $17,235-239$.

J. Fan (1991a). On the optimal rates of convergence for nonparametric deconvolution problem. The Annals of Statistics, 19, 1257-1272.

J. Fan (1991b). Asymptotic normality for deconvolution kernel estimators, Sankhya Series A, 53, 97-110.

J. Fan \& J.-Y. Koo (2002). Wavelet deconvolution. IEEE Transactions on Information Theory, 48, $734-747$.

C. Hesse (1999) Data-driven deconvolution. Journal of Nonparametric Statistics, 10, 343-373.

I.A. Ibragimov \& R.Z. Hasminskii (1983). Estimation of distribution density. Journal of Soviet Mathematics, 21, 40-57.

J.-Y Koo (1999). Logspline deconvolution in Besov space. Scandinavian Journal of Statistics, 26, 73-86.

M.C. Liu \& R.L. Taylor (1989). A consistent nonparametric density estimator for the deconvolution problem. The Canadian Journal of Statistics, 17, 427-438.

E. Masry (1991). Multivariate probability density deconvolution for stationary random processes. IEEE Transactions on Information Theory, 37, 1105-1115.

A. Meister (2004). On the effect of misspecifying the error density in a deconvolution problem. The Canadian Journal of Statistics, 32(4), 439-449.

Y. Meyer (1990). Ondelettes et opérateurs, Tome I, Hermann.

M. Pensky (2002). Density deconvolution based on wavelets with bounded supports. Statistics and Probability Letters, 56, 261-269.

M. Pensky \& B. Vidakovic (1999). Adaptive wavelet estimator for nonparametric density deconvolution. The Annals of Statistics, 27, 2033-2053.

L. Stefanski \& R.J. Carroll (1990). Deconvoluting kernel density estimators. Statistics, 21, 1696-184.

M. Talagrand (1996). New concentration inequalities in product spaces. Inventiones Mathematicae, 126, $505-563$. 
A.B. Tsybakov (2000). On the best rate of adaptive estimation in some inverse problems. ComptesRendus de l'Académie des Sciences, Paris, Série I Mathématiques, 330, 835-840.

C.H. Zhang (1990). Fourier methods for estimating mixing densities and distributions. The Annals of Statistics, 18, 806-831. 
Fabienne COMTE: fabienne.comte@univ-paris5.fr Université René Descartes-Paris 5 45, rue des Saint-Pères 75270 Paris Cedex 06 FRANCE

Yves ROZENHOLC: yves.rozenholc@math-info.univ-paris5.fr Laboratoire MAP5, UMR 5145 Université René Descartes-Paris 5 45, rue des Saint-Pères 75270 Paris Cedex 06 FRANCE

Marie-Luce TAUPIN: marie-luce.taupin@math.u-psud.fr Département de mathématiques, UMRC 8628

Université Paris-Sud 91405 Orsay FRANCE 